\title{
An algebraic subgrid scale finite element method for the convected Helmholtz equation in two dimensions with applications in aeroacoustics
}

\author{
Oriol Guasch $^{\mathrm{a}, *}$, Ramon Codina ${ }^{\mathrm{b}}$ \\ ${ }^{a}$ ICR, Enginyeria pel Control del Soroll, C/Berruguete 52 (Vila Olimpica-Vall d'Hebron), 08035 Barcelona, Catalonia, Spain \\ ${ }^{\mathrm{b}}$ Universitat Politècnica de Catalunya, Jordi Girona 1-3, Edifici C1, 08034 Barcelona, Catalonia, Spain
}

Received 17 October 2006; received in revised form 31 May 2007; accepted 3 June 2007

Available online 14 June 2007

\begin{abstract}
An algebraic subgrid scale finite element method formally equivalent to the Galerkin Least-Squares method is presented to improve the accuracy of the Galerkin finite element solution to the two-dimensional convected Helmholtz equation. A stabilizing term has been added to the discrete weak formulation containing a stabilization parameter whose value turns to be the key for the good performance of the method. An appropriate value for this parameter has been obtained by means of a dispersion analysis. As an application, we have considered the case of aerodynamic sound radiated by incompressible flow past a two-dimensional cylinder. Following Lighthill's acoustic analogy, we have used the time Fourier transform of the double divergence of the Reynolds stress tensor as a source term for the Helmholtz and convected Helmholtz equations and showed the benefits of using the subgrid scale stabilization.
\end{abstract}

(C) 2007 Elsevier B.V. All rights reserved.

Keywords: Convected Helmholtz equation; Convected wave equation; Aeroacoustics; Subgrid scale stabilization; Aerodynamic sound

\section{Introduction}

Acoustic waves propagating in a stationary background media are solutions of the well-known wave equation. Acoustic waves are generated by sound sources, which may be considered as regions of space in contact with the fluid (or subregions in motion of the fluid itself) where energy of any origin is transformed into acoustic energy to be propagated outward as sound waves. The wave equation can be easily derived from the continuity and Euler equations for an isentropic flow, assuming quiescence and neglecting all non-linear terms. In the case of waves propagating in a flow with uniform mean speed, a convected wave equation can be derived that is valid for arbitrary values of the Mach number, up to transonic

\footnotetext{
${ }^{*}$ Corresponding author. Tel./fax: +34 934286339.

E-mail addresses: oguasch@icrsl.com (O. Guasch), ramon.codina@ upc.edu (R. Codina).
}

flows (see e.g., [17,26] and references therein). The convected wave equation becomes of importance in many practical problems in aeroacoustics involving aerodynamic sound generated by aircraft engine fans and compressors $[17,26]$.

Just as significant as the wave equation is its time Fourier transform: the Helmholtz equation, which gives the spatial distribution of the acoustic field for a given wavenumber. Analogously, the time Fourier transform of the convected wave equation describes the spatial distribution of acoustic waves propagating in a background uniform flow. This equation, known as the time-reduced version of the convected wave equation, will be hereafter referred to as the convected Helmholtz equation. It is the main purpose of this paper to solve the convected Helmholtz equation for a background flow of constant speed, in the framework of finite element methods (FEM).

A large amount of work has been carried out to find non-polluted numerical solutions to the Helmholtz 
equation using finite element methods. The pollution error stems from the fact that the weak form associated to the Helmholtz equation is not positive definite for large wavenumbers, although it satisfies a Gårding inequality that allows Galerkin methods to be applied to it [29]. However, the inf-sup constant has an inverse dependence with the wavenumber $k$ that leads to a deterioration of the stability and to the appearance of the pollution error for large values of $k$. A dispersion analysis of the weak form interior numerical stencil reveals that this error is related to the fact that discrete waves propagate with a discrete wavenumber, $k_{h}$, instead of the continuous one. The difference between both wavenumbers, $k-k_{h}$, increases for large $k$ 's and a phase error appears in the numerically solved waves (see e.g., $[21,22,3])$.

In order to avoid this problem several methods have been developed, some of them in the more general context of the CDR (convection-diffusion-reaction) equations. The basic idea of several of these methods is to add a stabilizing term to the discrete weak form of the problem that enhances the behaviour of the solution, diminishing the pollution effects. This is the case in $[21,22,46]$ where the GLS (Galerkin Least Squares) was applied to the Helm-

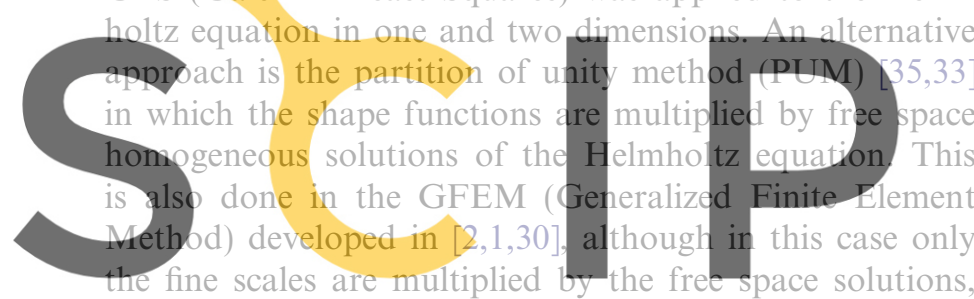
while standard shape functions are used for the coarse

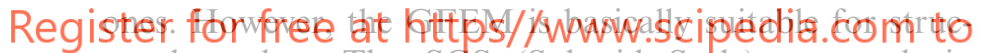
tured meshes. The SGS (Subgrid Scale) approach in $[27,28]$ was applied to the Helmholtz equation using different models for the subscales [4,37] and motivated the inclusion of the element boundary residues into the weak form [39]. Other approaches to find improved numerical solutions for the Helmholtz equation have considered the use of bubble functions [13] or enriching the standard polynomial field by means of plane waves. In the discontinuous enrichment method (DEM) [12] standard shape functions are used for the coarse scales while free space homogeneous solutions are added to them representing the influence of the finer scales. In $[38,10]$ comparisons of the performance of some of these methods can be found and in [20] a recent and complete review of several finite element methods for time harmonic acoustics is provided.

In contrast with the Helmholtz equation and as far as we know, the convected Helmholtz equation has received much less attention. In [25], some stabilizing methods for the CDR scalar equation were readapted to include the case of a production source term and, recently, an analysis of the accuracy of the Galerkin solution to the convected wave equation has been addressed in $[15,16]$. Dispersion and amplitude errors for upstream and downstream propagating waves using different finite element types have been studied as well as their dependence on several parameters such as Mach number magnitude and wave and flow orientations.

In this paper, an algebraic subgrid scale finite element method for the convected Helmholtz equation is proposed. A stabilizing term is added to the discrete weak form of the problem containing a stabilization parameter that is determined by means of a dispersion analysis. As we will deal with selfadjoint operators, the herein proposed method coincides with the GLS approach to the problem except for a minus sign that can be included in the definition of the stabilization parameter [20,5]. The GLS approach is probably the cheapest and simplest way to provide stabilization to the Helmholtz equation because its implementation is made at almost no computational cost [20]. In [10], it was concluded that the GLS performed superior to residual-free bubbles (only effective in one dimension) but that the QSFEM (Quasi Stabilized FEM) or increasing the degree of the polynomial field were clearly better in lowering the dispersion error. However, the QSFEM is rather difficult to adapt to non-uniform meshes and irregular boundary conditions, as already quoted in [10]. Some of the methods cited in the above paragraphs can also yield better results than GLS but usually at the cost of more

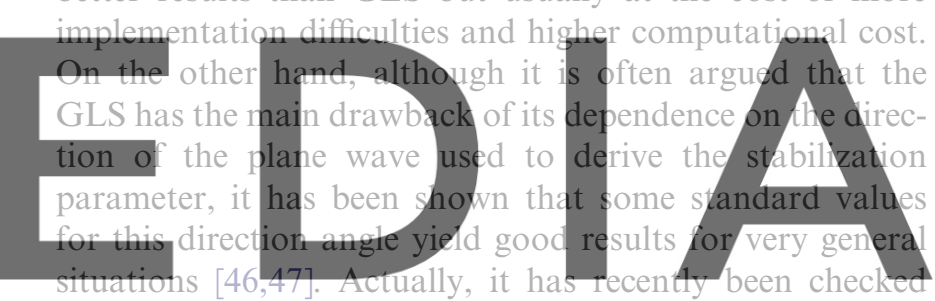

by means of numerical experiments that the GLS clearly

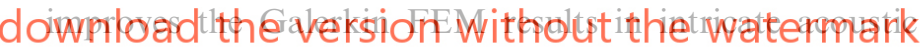
fields such as e.g., the noise radiation in an automotive interior or the scattering from a submarine-shaped obstacle [23]. In addition, the stabilization parameters usually derived for a particular mesh (e.g., a mesh of bilinear elements) still work when changing to unstructured meshes $[23,45]$. As a consequence, we may conclude that GLS still becomes an appealing option when considering its easy implementation aspect and low computational cost, together with the clear improvement of the standard Galerkin FEM results. It is worthwhile to mention that recent work on GLS has involved adapting the stabilization parameter for triangular and distorted elements [24,32].

The work presented in this paper for the convected Helmholtz equation tends to confirm the general features found in the GLS application to the Helmholtz equation. A stabilization parameter is derived for a structured mesh of quadrilateral bilinear elements, which yields exact nodal values for a wave propagating at a given direction in a background uniform flow of constant speed. This parameter also performs well if we use, instead, a mesh of unstructured quadrilateral elements. We have decided not to work with many simple problems with analytical solution (the performance of the method in these cases could readily be outlined from the herein presented results, the analysis in $[15,16]$ and the GLS application to the Helmholtz 
equation) but to address a more intricate case in the line of what is done in [23]. The case of aerodynamic sound generated by flow past a two-dimensional cylinder has been considered using the Lighthill acoustic analogy approach [34]. A first incompressible computational fluid dynamic simulation (not to be detailed in this paper) has been performed in order to obtain the time evolution of the double divergence of the Reynolds stress. This quantity has been time Fourier transformed to the frequency domain and used as the acoustic source term for the Helmholtz and convected Helmholtz equation. Even though an unstructured mesh of triangular elements has been used in this example, the benefits of using the GLS stability parameter, derived from a quadrilateral bilinear element mesh, become apparent and the dispersion error in the outward generated waves is clearly reduced.

The paper is organized as follows. In Section 2 the relations among the wave equation, the Helmholtz equation and their convected counterparts are established. Fourier transform pairs together with Galilean and Lorentz transformations relate these equations. Change of variables by means of these transformations offer a first possibility to solve the convected Helmholtz equation. On the other hand, appropriate boundary conditions are also given for
the latter. In Section 3 we present the weak form of the
problem and the subgrid scale finite element method pro-
posed to solve it. The dispersion analysis to find the value
for the stabilization parameter is carried out. In Seetion 4
we present the numerical examples. We first show how
the method yields an exact nodal solution for a wave propagating in a mean flow of constant speed and we then pres-

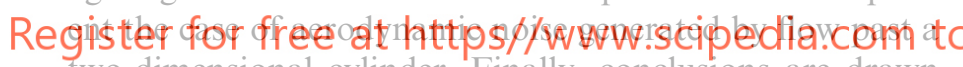
two-dimensional cylinder. Finally, conclusions are drawn in Section 5.

\section{Steady and convected wave and Helmholtz equations}

\subsection{Wave equation and convected wave equation}

The inhomogeneous wave equation for the propagation of pressure perturbations in a stationary, ideal medium is given by (acoustic wave equation)

$\left(c_{0}^{-2} \partial_{t t}^{2}-\nabla^{2}\right) p(\boldsymbol{x}, t)=s(\boldsymbol{x}, t)$,

where $p(\boldsymbol{x}, t)$ stands for the acoustic pressure, $s(\boldsymbol{x}, t)$ is the source term, $c_{0}$ is the sound speed in the medium and $\nabla^{2}$ represents, as usual, the Laplacian operator. Here and in the sequel we will denote by $\partial_{t}$ the partial time derivative $\partial / \partial t$, by $\partial_{t t}^{2}$ the partial time derivative of order two $\partial^{2} / \partial t^{2}$, by $\partial_{i}$ the spatial partial derivative $\partial / \partial x_{i}, \mathrm{i}=1 \ldots n_{s d}$, and by $\partial_{i j}^{2}$ the spatial partial derivatives up to order two $\partial^{2} / \partial x_{i} \partial x_{j}, i, j=1 \ldots n_{s d} . n_{s d}$ denotes the space dimension and we will take $n_{s d}=3$ in the exposition. As usual, we will also identify $x_{1} \equiv x, x_{2} \equiv y$ and $x_{3} \equiv z$. The classical summation convention will be adopted for repeated indices.

For acoustic waves propagating in an homentropic, irrotational flow, (1) is no longer valid and has to be replaced by a linear equation for the time derivative of the velocity potential or for the perturbation velocity potential [26]. However, in the special case of a flow with mean velocity $\boldsymbol{U}_{0}(\boldsymbol{x})$ at a low Mach number $\left(M^{2} \ll 1, M=\|\boldsymbol{M}\|, \boldsymbol{M}(\boldsymbol{x}):=\boldsymbol{U}_{0}(\boldsymbol{x}) / c_{0}\right)$ variations in the mean density and sound speed can be neglected and the acoustic wave propagation can be described by a relatively simple equation, namely the convected wave equation

$\left[c_{0}^{-2}\left(\partial_{t}+\boldsymbol{U}_{0}(\boldsymbol{x}) \cdot \nabla\right)^{2}-\nabla^{2}\right] p(\boldsymbol{x}, t)=s(\boldsymbol{x}, t)$.

\section{Remarks}

- If $\boldsymbol{U}_{0}$ is constant (uniform flow) (2) becomes valid for Mach numbers up to one (transonic flows). Then, Eqs. (1) and (2) become equivalent as they are related by a simple Galilean transformation of the coordinatesystem

$x^{\prime}=x+U_{0} t$.

Uniform flow will be assumed in what follows throughout the paper.

- It is quite customary to use the material derivative

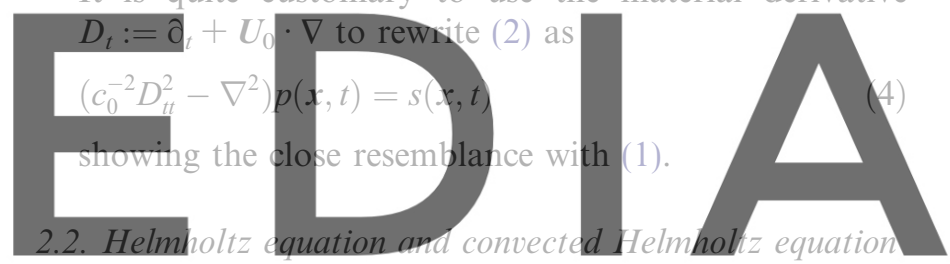

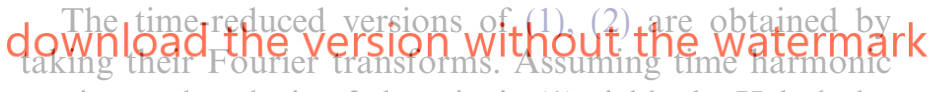

motion and replacing $\partial_{t}$ by -iw in (1) yields the Helmholtz equation

$\left(-\nabla^{2}-k_{0}^{2}\right) \hat{p}(\boldsymbol{x}, \omega)=\hat{s}(\boldsymbol{x}, \omega)$,

with $k_{0}=\omega / c_{0}$ being the wavenumber and $\omega$ the radian frequency. Analogously, replacing $\partial_{t}$ by $-\mathrm{i} \omega$ in (2) yields the convected wave equation

$-\left[\nabla^{2}+\left(k_{0}+\mathrm{i} \boldsymbol{M} \cdot \nabla\right)^{2}\right] \hat{p}(\boldsymbol{x}, \omega)=\hat{s}(\boldsymbol{x}, \omega)$,

with $\mathrm{i}=\sqrt{-1}$. We identify, for subsequent sections, the Helmholtz and convected Helmholtz differential operators as

$$
\begin{aligned}
\mathscr{L}_{\mathrm{H}} & :=\left(-\nabla^{2}-k_{0}^{2}\right) \\
\mathscr{L}_{\mathrm{CH}} & :=-\left[\nabla^{2}+\left(k_{0}+\mathrm{i} \boldsymbol{M} \cdot \nabla\right)^{2}\right] \\
& =-\nabla^{2}-k_{0}^{2}-2 \mathrm{i} k_{0} \boldsymbol{M} \cdot \nabla+(\boldsymbol{M} \cdot \nabla)(\boldsymbol{M} \cdot \nabla) \\
& =-\nabla \cdot\left(2 \mathrm{i} k_{0} \boldsymbol{M} \hat{p}\right)-\nabla \cdot\{[\boldsymbol{I}-(\boldsymbol{M} \otimes \boldsymbol{M})] \cdot \nabla \hat{p}\}-k_{0}^{2} \hat{p},
\end{aligned}
$$

where in the last line of (8) we have used the fact that $\boldsymbol{M}$ is constant and $\boldsymbol{I}$ stands for the identity. From (7) and (8) it is quite straightforward to show that both, the Helmholtz equation and the convected Helmholtz equation 
correspond to particular cases of the more general Convection-Diffusion-reaction (CDR) equation. On the other hand, note that disregarding the boundary condition contributions, as it is common practice in the present numerical context, both operators (7) and (8) are selfadjoint i.e.,

$\mathscr{L}_{\mathrm{H}}^{\dagger}=\mathscr{L}_{\mathrm{H}}, \quad \mathscr{L}_{\mathrm{CH}}^{\dagger}=\mathscr{L}_{\mathrm{CH}}$.

As for (1), (2), Eqs. (5) and (6) can also be related but now via a full Lorentz transformation (also known as a Prandtl-Glauert transformation in the aerodynamic context). This offers a first possibility to solve the convected wave equation by first converting it in the more simple Helmholtz equation, then solving for it and finally reverting to the original variables (see Fig. 1 for a schematic representation of the relations among all Eqs. (1), (2), (5) and (6)). The full Lorentz transformation relating (5), (6) involves a rotation plus a boost in the $x$-direction. The two changes will be briefly and independently presented for clarity and given in a compact form at the end. Rotation: Let us denote by $\boldsymbol{R}$ the rotation matrix that transforms the Mach number vector $\boldsymbol{M}$ so that it only has $x$-component in the new coordinate-system $x^{\prime}$, i.e., $\mathbb{M}^{\prime}=\boldsymbol{R} \boldsymbol{M}$ with $\boldsymbol{M}^{\prime}=(M, 0,0)^{\mathrm{T}}$. If we apply the change

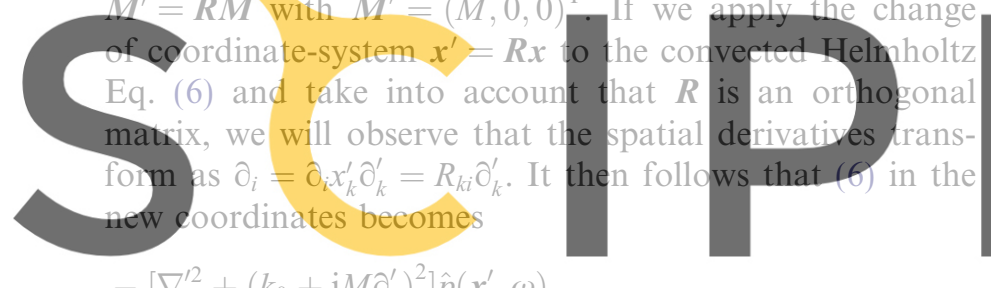

$-\left[\nabla^{\prime 2}+\left(k_{0}+\mathrm{i} M \partial_{*}^{\prime}\right)^{2}\right] \hat{p}\left(x^{\prime}, \omega\right)$

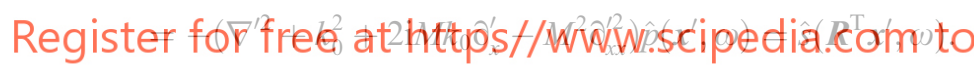

Boost in the $x$-direction: We next perform a boost in the $x$-direction to $(10)$ consisting in taking $\boldsymbol{x}^{\prime \prime}=\boldsymbol{D} \boldsymbol{x}^{\prime}$ with $\boldsymbol{D}$ being the diagonal matrix $\boldsymbol{D}=\operatorname{diag}\left(\beta^{-1}, 1,1\right)$ and $\beta$ being defined as usual by $\beta:=\sqrt{1-M^{2}}$. We also take $k_{0}^{\prime \prime}=\beta^{-1} k_{0}$ and $\hat{p}^{\prime \prime}\left(\boldsymbol{x}^{\prime \prime}, k_{0}^{\prime \prime}\right)=\hat{p}\left(\boldsymbol{x}^{\prime}, k_{0}\right) \exp \left(\mathrm{i} k_{0}^{\prime \prime} M x^{\prime \prime}\right)$. Again, after some straightforward algebra we can obtain an expression for (10) in the new coordinates $\boldsymbol{x}^{\prime \prime}$ :

$\left(\nabla^{\prime \prime 2}+k_{0}^{\prime \prime 2}\right) \hat{p}^{\prime \prime}\left(\boldsymbol{x}^{\prime \prime}, \omega\right)=\mathrm{e}^{\left(i k_{0}^{\prime \prime} M x^{\prime \prime}\right)} \hat{s}\left(\boldsymbol{R}^{\mathrm{T}} \boldsymbol{D}^{-1} \boldsymbol{x}^{\prime \prime}, \beta k_{0}^{\prime \prime}\right)$.

\section{Remarks}

- Eq. (11) is a Helmholtz equation with a source term modified by an exponential factor. As the source term is a known input quantity for the problem, it will have to be expressed in terms of the new coordinates $\boldsymbol{x}^{\prime \prime}$ to solve (11).

- The full Lorentz transformation can be set in a compact form as

$$
\begin{aligned}
& \boldsymbol{x}^{\prime \prime}=\boldsymbol{D} \boldsymbol{R} \boldsymbol{x}, \quad k_{0}^{\prime \prime}=\beta^{-1} k_{0}, \\
& \hat{p}^{\prime \prime}\left(\boldsymbol{x}^{\prime \prime}, k_{0}^{\prime \prime} \omega\right)=\hat{p}(\boldsymbol{R} \boldsymbol{x}, \omega) \exp \left[\mathrm{i} \beta^{-1} k_{0}(\boldsymbol{R M}) \cdot(\boldsymbol{D} \boldsymbol{R} \boldsymbol{x})\right] .
\end{aligned}
$$

\subsection{Boundary conditions for the convected Helmholtz problem and strong formulation}

When solving (11) in an open domain $\Omega_{\text {ac }}^{\prime \prime}$, it has to be completed with appropriate boundary conditions on $\partial \Omega_{\mathrm{ac}}^{\prime \prime}$. Considering Dirichlet, Neumann and Sommerfeld conditions we are left with the strong or differential form of the Helmholtz problem: find the acoustic pressure $\hat{p}^{\prime \prime}: \Omega_{\mathrm{ac}}^{\prime \prime} \mapsto \mathbb{C}$, being $\Omega_{\mathrm{ac}}^{\prime \prime} \subset \mathbb{R}^{d}$ a bounded domain with

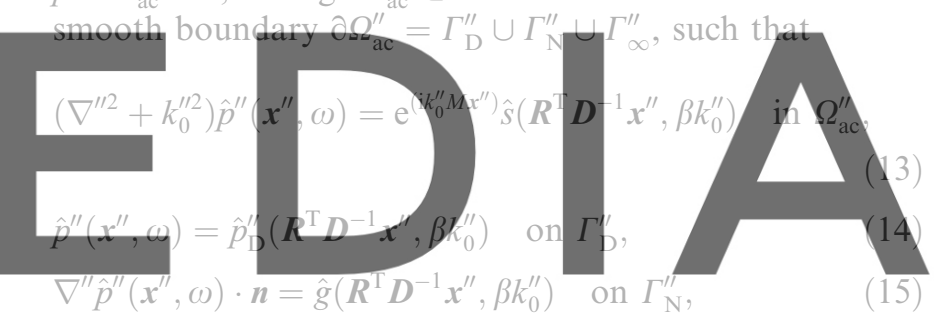

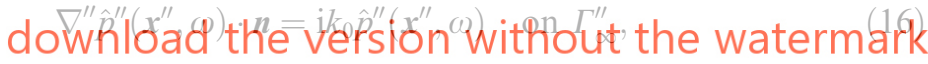

where $n$ represents the normal pointing outwards of $\Gamma_{\mathrm{N}}^{\prime \prime}$ and $\Gamma_{\infty}^{\prime \prime}$ and $\hat{g}: \Gamma_{\mathrm{N}}^{\prime \prime} \mapsto \mathbb{C}$ is prescribed on $\Gamma_{\mathrm{N}}^{\prime \prime}$.

As earlier mentioned, solving (13) and reverting to the original coordinate-system and variables is a suitable option to find the solution of the convected Helmholtz equation. However, if we are interested in directly solving this equation we will need appropriate boundary conditions for it. These conditions can be found by reverting the Helmholtz equation boundary conditions (14)-(16) to the original variables and coordinate-system through the

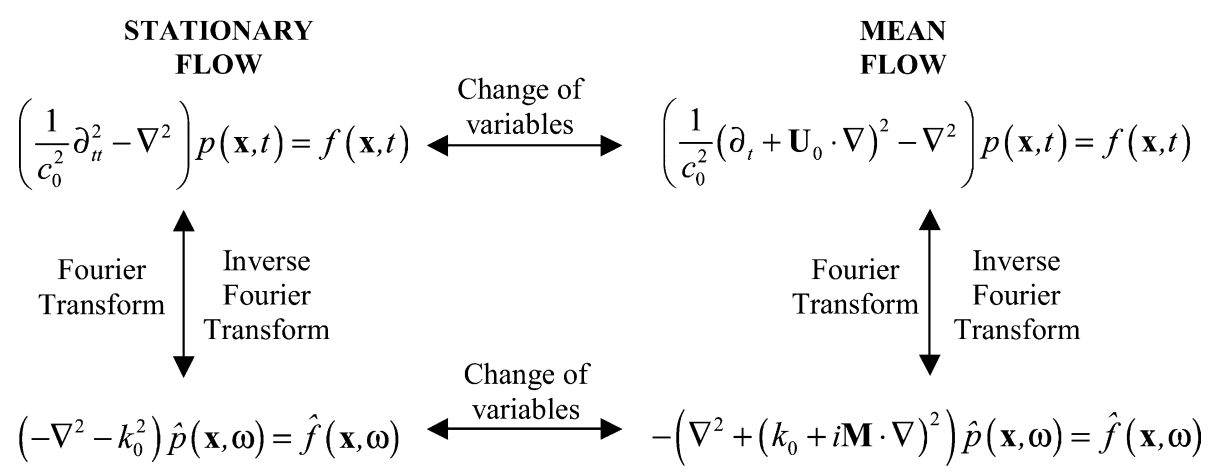

Fig. 1. Equations framework. 
Lorentz transformation (12). This yields the following results:

Sommerfeld's radiation condition: The Sommerfeld's radiation condition for the convected Helmholtz equation becomes

$\nabla \hat{p}(\boldsymbol{x}, \omega) \cdot \boldsymbol{n}=\mathrm{i} k_{0} \alpha_{S} \hat{p}(\boldsymbol{x}, \omega)$

$\alpha_{S}:=\beta^{-1}\left[1-M \beta^{-1}(\boldsymbol{n} \cdot \overline{\boldsymbol{M}})\right]$,

$\overline{\boldsymbol{M}}$ being the normalized Mach number vector, $\overline{\boldsymbol{M}}=(1 / M) \boldsymbol{M}$.

Neumann boundary condition: The Neumann boundary condition for the convected Helmholtz equation becomes

$\nabla \hat{p}(\boldsymbol{x}, \omega) \cdot \boldsymbol{n}=-\mathrm{i} k_{0} \alpha_{N} \hat{p}(\boldsymbol{x}, \omega)+\hat{g}\left(\boldsymbol{x}, k_{0}\right)$,

$\alpha_{N}:=\beta^{-2}(\boldsymbol{n} \cdot \overline{\boldsymbol{M}})=\beta^{-1}-\alpha_{S}$.

Note that, as expected $\alpha_{S}=1, \alpha_{N}=0$ for $M=0$.

Taking into account (17)-(20), the strong formulation for the convected Helmholtz equation problem can be written as: find the acoustic pressure $\hat{p}: \Omega_{\mathrm{ac}} \mapsto \mathbb{C}$, being $\Omega_{\mathrm{ac}} \subset \mathbb{R}^{d}$ a bounded domain with smooth boundary $\partial \Omega_{\mathrm{ac}}=\Gamma_{\mathrm{D}} \cup$ $\Gamma_{\mathrm{N}} \cup \Gamma_{\infty}$, such that

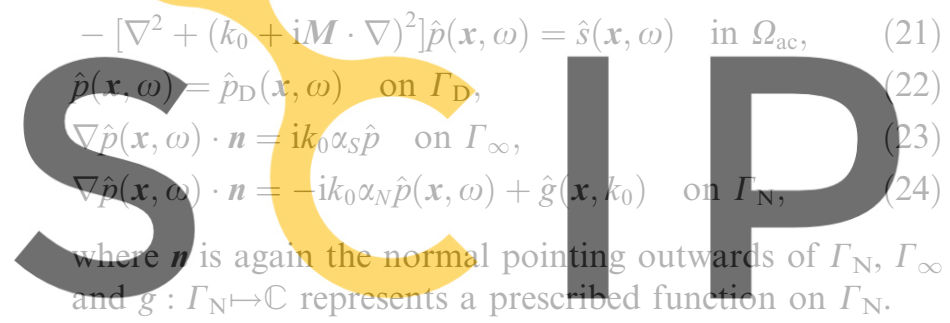

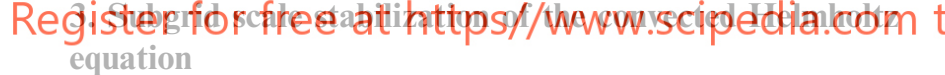

\subsection{Continuous weak form}

In order to solve the differential convected Helmholtz equation by means of a finite element method we first have to obtain its continuous weak form. For this purpose, let us first introduce the following functional spaces

$$
\begin{aligned}
& \mathscr{W}:=\left\{\hat{p}(\boldsymbol{x}) \in H^{1}(\Omega) ; \hat{p}(\boldsymbol{x})=\hat{p}(\boldsymbol{x})_{\mathrm{D}} \text { on } \Gamma_{\mathrm{D}}\right\} \\
& \mathscr{W}_{0}:=\left\{q(\boldsymbol{x}) \in H^{1}(\Omega) ; q=0 \text { on } \Gamma_{\mathrm{D}}\right\}
\end{aligned}
$$

where $H^{1}(\Omega)$ denotes as usual the first-order Sobolev space of functions with square integrable derivatives of order up to one. We also introduce the following notation: the scalar products in $L^{2}(\Omega)$ and $L^{2}(\Gamma)$ will be denoted by

$(v, w)_{\Omega}:=\int_{\Omega} w^{*} v \mathrm{~d} \Omega \quad(v, w)_{\Gamma}:=\int_{\Gamma} w^{*} v \mathrm{~d} \Gamma$,

with $*$ standing for the complex conjugate. The duality pairing between $H_{0}^{1}(\Omega)$ and $H^{-1}(\Omega)$ will be represented by $\langle\cdot, \cdot\rangle_{\Omega}$.

The weak form corresponding to the convected Helmholtz Eq. (21) with boundary conditions (22)-(24) can be stated as: find $\hat{p} \in \mathscr{W}$ such that $a(\hat{p}, q)=l(q) \quad \forall q \in \mathscr{W}_{0}$,

where $a(\hat{p}, q)$ stands for the sesquilinear form

$$
\begin{aligned}
a(\hat{p}, q):= & -2 \mathrm{i} k_{0} M_{j}\left(\partial_{j} \hat{p}, q\right)_{\Omega_{\mathrm{ac}}}+\left[\delta_{i j}-M_{i} M_{j}\right]\left(\partial_{i} \hat{p}, \partial_{j} q\right)_{\Omega_{\mathrm{ac}}} \\
& -k_{0}^{2}(\hat{p}, q)_{\Omega_{\mathrm{ac}}}-\mathrm{i} k_{0} \alpha_{S}(\hat{p}, q)_{\Gamma_{\infty}}+\mathrm{i} k_{0} \alpha_{N}(\hat{p}, q)_{\Gamma_{\mathrm{N}}}
\end{aligned}
$$

and $l(q)$ is the antilinear functional

$l(q)=\left\langle\hat{s}_{h}, q\right\rangle_{\Omega_{\mathrm{ac}}}+(\hat{g}, q)_{\Gamma_{\mathrm{N}}}$,

where we have considered $\hat{g}$ in $L^{2}\left(\Gamma_{\mathrm{N}}\right)$.

\subsection{Discrete weak form}

Given a partition of $\Omega_{\mathrm{ac}}$ into finite elements $\left\{\Omega^{e}\right\}_{e=1}^{n_{e}}$ and the finite-dimensional subspaces $\mathscr{W}_{h} \subset \mathscr{W}, \mathscr{W}_{0, h} \subset \mathscr{W}_{0}$, the Galerkin finite element approach to problem (28) consists in finding $\hat{p}_{h} \in \mathscr{W}_{h}$ such that

$a\left(\hat{p}_{h}, q_{h}\right)=l\left(q_{h}\right) \quad \forall q_{h} \in \mathscr{W}_{0, h}$.

Note that by taking $q_{h}=\hat{p}_{h}$ and ignoring the boundary terms in the sesquilinear form we are left with the inequality

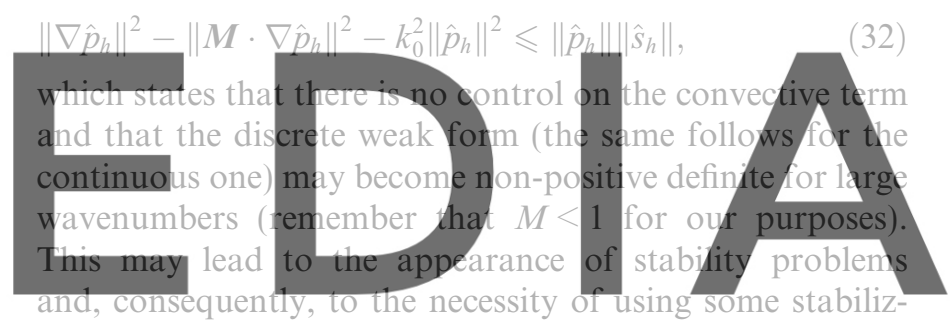

ing techniques to solve (31). In (32), $\|\cdot\|$ represents the downitolad the version without the watermark

\subsection{Subgrid scale stabilization}

To avoid the stability problems in (31), a subgrid scale (SGS) finite element method will be used $[27,28]$. The key idea of the SGS finite approach is to decompose the continuous spaces $\mathscr{W}=\mathscr{W}_{h} \oplus \widetilde{\mathscr{W}}, \mathscr{W}_{0}=\mathscr{W}_{0, h} \oplus \widetilde{\mathscr{W}}_{0}$, being $\widetilde{\mathscr{W}}$ and $\widetilde{\mathscr{W}}_{0}$ any continuous spaces to respectively complete $\mathscr{W}_{h}, \widetilde{\mathscr{W}}_{0}$ in $\mathscr{W}, \mathscr{W}_{0}$. Then, any function in $\mathscr{W}$ can be split as $\hat{p}=\hat{p}_{h}+\tilde{\hat{p}}, \hat{p}_{h}$ representing the part of $\hat{p}$ that can be captured with the finite element mesh and $\tilde{\hat{p}}$ the subscale or subgrid scale. Making the same decomposition for the test function and substituting into (31), we obtain the following two equations, respectively governing the large scales and subscales behavior

$a\left(\hat{p}_{h}, q_{h}\right)+a\left(\tilde{\hat{p}}, q_{h}\right)=l\left(q_{h}\right) \quad \forall q_{h} \in \mathscr{W}_{0, h}$,

$a\left(\hat{p}_{h}, \tilde{q}\right)+a(\tilde{\hat{p}}, \tilde{q})=l(\tilde{q}) \quad \forall \tilde{q} \in \widetilde{\mathscr{W}}_{0}$.

The goal consists in finding an approximate value for the subscales $\tilde{\hat{p}}$ (i.e., an approximate solution to (34)) and to substitute it into (33) to account for their effects on the large scales, resolvable by the finite element mesh. The various ways in how this can be done give place to different subgrid scale stabilizing methods. 
If the algebraic subgrid scale (ASGS) approach is followed, it can be shown that the large scale Eq. (33) is modified to (see e.g., [5])

$a\left(\hat{p}_{h}, q_{h}\right)+\left(-\mathscr{L}_{\mathrm{CH}}^{\dagger}\left(q_{h}\right), \tau_{\mathrm{SGS}}\left[\mathscr{L}_{\mathrm{CH}}\left(\hat{p}_{h}\right)-\hat{s}_{h}\right]\right)_{\bar{\Omega}_{\mathrm{ac}}}=l\left(q_{h}\right)$,

where $\mathscr{L}_{\mathrm{CH}}$ is the differential convected Helmholtz operator defined in (8) and $\tau_{\mathrm{SGS}}$ is a stabilization parameter to be determined below. The notation $(\cdot, \cdot)_{\bar{\Omega}_{\mathrm{ac}}}$ stands for

$$
(f, g)_{\bar{\Omega}_{\mathrm{ac}}}=\int_{\bar{\Omega}_{\mathrm{ac}}} g^{*} f, \quad \int_{\bar{\Omega}_{\mathrm{ac}}}:=\sum_{e=1}^{n_{e}} \int_{\Omega^{e}} .
$$

It is expected that the modified sesquilinear form (35) together with an appropriate choice for the stabilization parameter $\tau_{\mathrm{SGS}}$ will avoid some of the stability problems detected when solving the convected Helmholtz equation $[15,16]$.

If instead of the ASGS method the GLS one is used, it can be shown that (35) is modified to (see again [5]) $a\left(\hat{p}_{h}, q_{h}\right)+\left(\mathscr{L}_{\mathrm{CH}}\left(q_{h}\right), \tau_{\mathrm{GLS}}\left[\mathscr{L}_{\mathrm{CH}}\left(\hat{p}_{h}\right)-\hat{s}_{h}\right]\right)_{\bar{\Omega}_{\mathrm{ac}}}=l\left(q_{h}\right)$.

$(37)$

Taking into account that the convected Helmholtz differential operator is selfadjoint (9), it follows that both methods

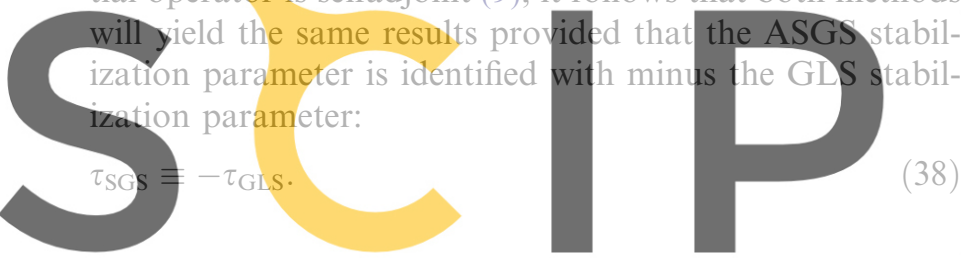

3.4. Stabilization parameter from a dispersion analysis

Register for free, at https/fwww. scipedia.com to

Helmholtz equation in two dimensions. A dispersion analysis will be performed considering a uniform mesh of element size $h \times h$ and the use of bilinear elements (see $[29,21,22,46])$. Let us first explicitly write the stabilized ASGS sesquilinear form (35) for the two-dimensional case without considering the boundary terms, as we will be interested in results concerning interior mesh nodes. This form is given by

$$
\begin{aligned}
a_{\mathrm{SGS}}\left(\hat{p}_{h}, w_{h}\right) & -2 \mathrm{i} k_{0} M_{x} \underbrace{\left(\partial_{x} \hat{p}_{h}, w_{h}\right)}_{C_{x}}-2 \mathrm{i} k_{0} M_{y} \underbrace{\left(\partial_{y} \hat{p}_{h}, w_{h}\right)}_{C_{y}} \\
& +\left[1-M_{x}^{2}\right] \underbrace{\left(\partial_{x} \hat{p}_{h}, \partial_{x} w_{h}\right)}_{D_{x x}}+\left[1-M_{y}^{2}\right] \underbrace{\left(\partial_{y} \hat{p}_{h}, \partial_{y} w_{h}\right)}_{D_{y y}} \\
& -2 M_{x} M_{y} \underbrace{\left(\partial_{x} \hat{p}_{h}, \partial_{y} w_{h}\right)}_{D_{x y}}-k_{0}^{2} \underbrace{\left(\hat{p}_{h}, w_{h}\right)}_{S} \\
& +\tau_{\mathrm{SGS}} 4 k_{0}^{2} M_{x}^{2} \sum_{n_{e}}\left(\partial_{x} \hat{p}_{h}, \partial_{x} w_{h}\right)_{\Omega_{e}} \\
& +\tau_{\mathrm{SGS}} 4 k_{0}^{2} M_{y}^{2} \sum_{n_{e}}\left(\partial_{y} \hat{p}_{h}, \partial_{y} w_{h}\right)_{\Omega_{e}} \\
& +\tau_{\mathrm{SGS}} 8 k_{0}^{2} M_{x} M_{y} \sum_{n_{e}}\left(\partial_{x} \hat{p}_{h}, \partial_{y} w_{h}\right)_{\Omega_{e}}-\tau_{\mathrm{SGS}} k_{0}^{4} \sum_{n_{e}}\left(\hat{p}_{h}, w_{h}\right)_{\Omega_{e}},
\end{aligned}
$$

where we have used the fact that $\left(\nabla \hat{p}_{h}, w_{h}\right)=-\left(\nabla w_{h}, \hat{p}_{h}\right)$ and we have identified the terms $C_{x}, C_{y}, D_{x x}, D_{y y}, D_{x y}$ and $S$ to simplify subsequent notation.

The nodal unknowns for interior nodes corresponding to the patch in Fig. 2 can be represented by the 9-point difference star

$$
\left[P_{a b}^{\mathrm{int}}\right]:=\left[\begin{array}{ccc}
\widehat{P}^{a-1, b+1} & \widehat{P}^{a, b+1} & \widehat{P}^{a+1, b+1} \\
\widehat{P}^{a-1, b} & \widehat{P}^{a, b} & \widehat{P}^{a+1, b} \\
\widehat{P}^{a-1, b-1} & \widehat{P}^{a, b-1} & \widehat{P}^{a+1, b-1}
\end{array}\right] .
$$

Analogously, when considering bilinear shape functions, the coefficients arising from $C_{x}, C_{y}, D_{x x}, D_{y y}, D_{x y}$ and $S$ can be written in compact form for the 9-point difference star in Fig. 2 as
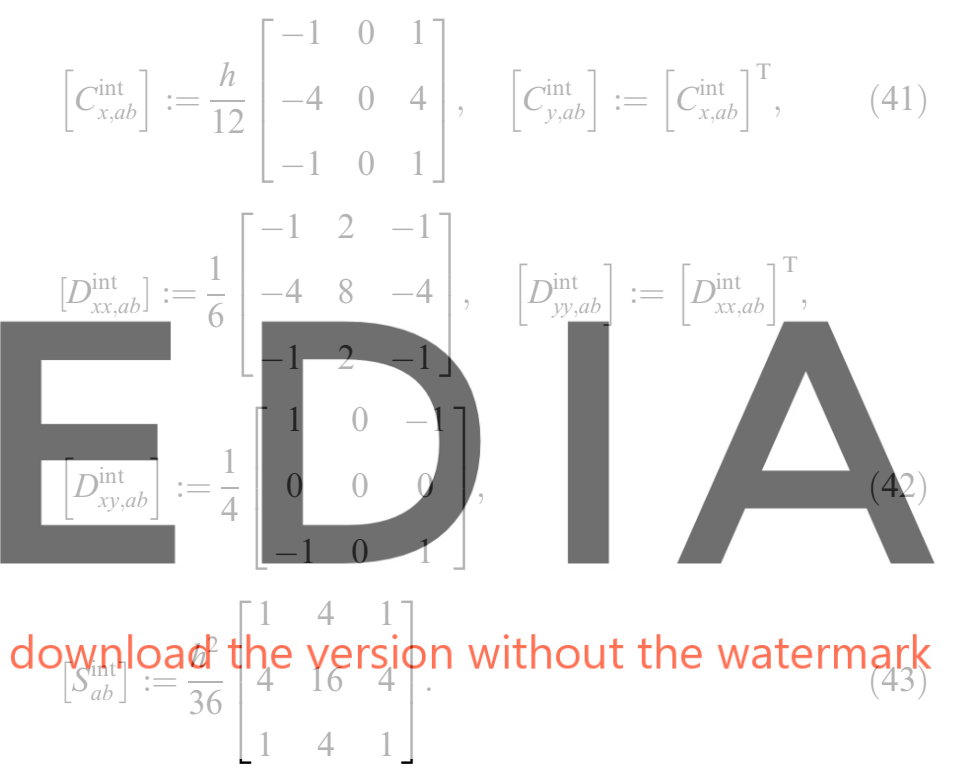

Taking into account the notation (40), (41), (10), (43), the equation for the interior node $a b$ (with no force acting on it) of the algebraic linear system associated to the discrete ASGS weak problem can be written as

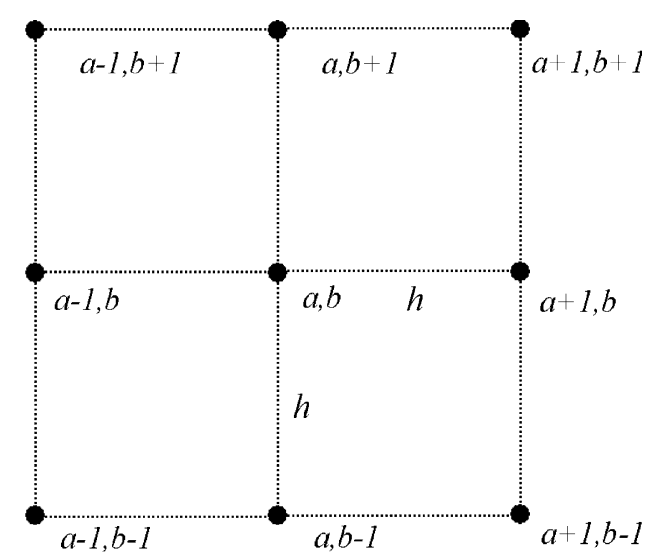

Fig. 2. Patch in a two-dimensional uniform mesh. 


$$
\begin{aligned}
-2 \mathrm{i} k_{0} M_{x}\left[C_{x, a b}^{\mathrm{int}}\right] & :\left[P_{a b}^{\mathrm{int}}\right]-2 \mathrm{i} k_{0} M_{y}\left[C_{x, a b}^{\mathrm{int}}\right]:\left[P_{a b}^{\mathrm{int}}\right] \\
& +\left(1-M_{x}^{2}\right)\left[D_{x x, a b}^{\mathrm{int}}\right]:\left[P_{a b}^{\mathrm{int}}\right] \\
& +\left(1-M_{y}^{2}\right)\left[D_{y y, a b}^{\mathrm{int}}\right]:\left[P_{a b}^{\mathrm{int}}\right] \\
& -2 M_{x} M_{y}\left[D_{x y, a b}^{\mathrm{int}}\right]:\left[P_{a b}^{\mathrm{int}}\right] \\
& -k_{0}^{2}\left[S_{a b}^{\mathrm{int}}\right]:\left[P_{a b}^{\mathrm{int}}\right] \\
& +\tau_{\mathrm{SGS}} 4 k_{0}^{2} M_{x}^{2}\left[D_{x x, a b}^{\mathrm{int}}\right]:\left[P_{a b}^{\mathrm{int}}\right] \\
& +\tau_{\mathrm{SGS}} 4 k_{0}^{2} M_{y}^{2}\left[D_{y y, a b}^{\mathrm{int}}\right]:\left[P_{a b}^{\mathrm{int}}\right] \\
& +\tau_{\mathrm{SGS}} 8 k_{0}^{2} M_{x} M_{y}\left[D_{x y, a b}^{\mathrm{int}}\right]:\left[P_{a b}^{\mathrm{int}}\right] \\
& -\tau_{\mathrm{SGS}} k_{0}^{4}\left[S_{a b}^{\mathrm{int}}\right]:\left[P_{a b}^{\mathrm{int}}\right]=0
\end{aligned}
$$

where : stands for a double contraction. If we now assume a plane wave solution with an effective wavenumber vector $\boldsymbol{k}_{0}^{\mathrm{eff}}=\left(k_{0 x}^{\mathrm{eff}}, k_{0 y}^{\mathrm{eff}}\right)$ (to be determined lately in this section) so that at node $m n$

\section{$\hat{p}^{m n}=\exp \left[\mathrm{i}\left(k_{0 \mathrm{x}}^{\mathrm{eff}} h m+k_{0 y}^{\mathrm{eff}} h n\right)\right]$}

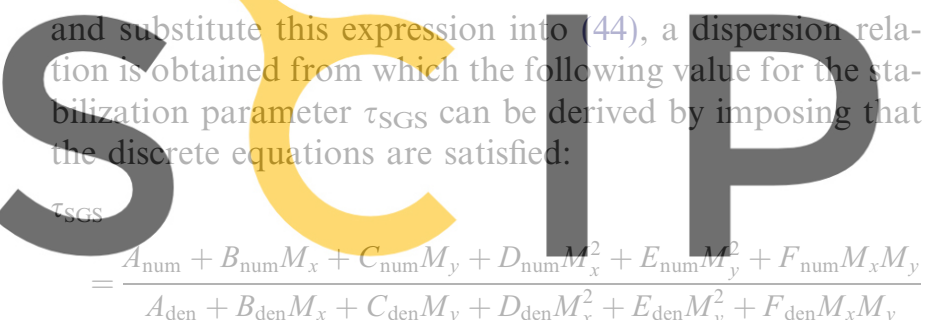

Register for free at https//www.scipedia.combto

with

$$
\begin{aligned}
A_{\text {num }}= & -\frac{2}{3}\left[4-\cos \left(k_{0 x}^{\mathrm{eff}} h\right)-\cos \left(k_{0 y}^{\mathrm{eff}} h\right)\right. \\
& \left.-2 \cos \left(k_{0 x}^{\mathrm{eff}} h\right) \cos \left(k_{0 y}^{\mathrm{eff}}\right)\right]+\frac{k_{0}^{2} h^{2}}{9}\left\{\left[2+\cos \left(k_{0 x}^{\mathrm{eff}} h\right)\right]\right. \\
& \left.\times\left[2+\cos \left(k_{0 y}^{\mathrm{eff}} h\right)\right]\right\} \\
B_{\text {num }}= & -\frac{2}{3} k_{0} h\left[\sin \left(k_{0 x}^{\mathrm{eff}} h\right) \cos \left(k_{0 y}^{\mathrm{eff}} h\right)+2 \sin \left(k_{0 x}^{\mathrm{eff}} h\right)\right] \\
C_{\text {num }}= & -\frac{2}{3} k_{0} h\left[\sin \left(k_{0 y}^{\mathrm{eff}} h\right) \cos \left(k_{0 x}^{\mathrm{eff}} h\right)+2 \sin \left(k_{0 y}^{\mathrm{eff}} h\right)\right]
\end{aligned}
$$

$D_{\text {num }}=\frac{2}{3}\left\{2-\cos \left(k_{0 x}^{\mathrm{eff}} h\right)\left[2+\cos \left(k_{0 y}^{\mathrm{eff}} h\right)\right]+\cos \left(k_{0 y}^{\mathrm{eff}} h\right)\right\}$,

$$
E_{\text {num }}=\frac{2}{3}\left\{2-\cos \left(k_{0 y}^{\mathrm{eff}} h\right)\left[2+\cos \left(k_{0 x}^{\mathrm{eff}} h\right)\right]+\cos \left(k_{0 y}^{\mathrm{eff}} h\right)\right\},
$$

$$
F_{\text {num }}=2 \sin \left(k_{0 x}^{\mathrm{eff}} h\right) \sin \left(k_{0 y}^{\mathrm{eff}} h\right)
$$

and

$$
\begin{aligned}
& A_{\text {den }}=-\frac{k_{0}^{4} h^{2}}{3}\left[2+\cos \left(k_{0 x}^{\mathrm{eff}} h\right)\right]\left[2+\cos \left(k_{0 y}^{\mathrm{eff}} h\right)\right], \\
& B_{\mathrm{den}}=0 \\
& C_{\text {den }}=0 \\
& D_{\text {den }}=4 k_{0}^{2} D_{\text {num }}, \\
& E_{\text {den }}=4 k_{0}^{2} E_{\text {num }}, \\
& F_{\text {den }}=4 k_{0}^{2} F_{\text {num }} .
\end{aligned}
$$

It remains now to find the appropriate value for the effective wavenumber vector in (47)-(58). This can be done in a quite straightforward manner by taking into account the results from Section 2. Our obiective is to find the effective wavenumber for a plane wave propagating at an arbitrary direction, say angle $\theta$ with the $x$-axis, in a uniform flow characterized by a Mach vector $\boldsymbol{M}$ being at an angle $\varphi$ with the $x$-axis (see Fig. 3). All that we have to do is to revert the full Lorentz transformation (12) for a plane wave

$\hat{p}^{\prime \prime}\left(\boldsymbol{x}^{\prime \prime}, \boldsymbol{k}_{0}^{\prime \prime}\right)=\exp \left(\mathrm{i} \boldsymbol{k}_{0}^{\prime \prime} \cdot \boldsymbol{x}^{\prime \prime}\right)$.

Hence, the plane wave propagating in the uniform medium

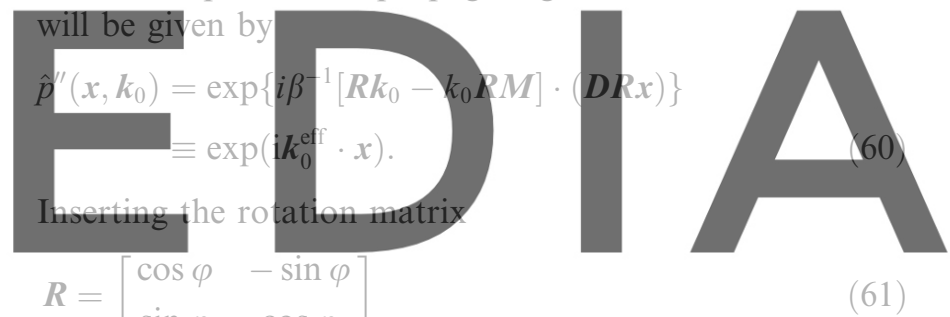

download the version without the watermark in (60) we arrive to the following values for the components of the effective wavenumber vector defined in the last equality of (60)

$k_{0 x}^{\mathrm{eff}}=k_{0} \beta^{-2}\left[\cos (\theta-\varphi) \cos \varphi-M_{x}\right]-k_{0} \beta^{-1} \sin (\theta-\varphi) \sin \varphi$,

$k_{0 y}^{\mathrm{eff}}=k_{0} \beta^{-2}\left[\cos (\theta-\varphi) \sin \varphi+M_{y}\right]-k_{0} \beta^{-1} \sin (\theta-\varphi) \cos \varphi$.

Note that $\boldsymbol{k}_{0}^{\text {eff }}$ is nothing but $\boldsymbol{k}_{0}^{\prime \prime}$ expressed in the original coordinates and variables. Expressions (62) and (63) are finally to be inserted in Eqs. (47)-(58) to find the appropriate value for the parameter $\tau_{\mathrm{SGS}}$.

\section{Remarks}

- It should be noted from the above formulation that while the flow orientation $\varphi$ is a given parameter, the plane wave orientation $\theta$ is an artifact of the analysis whose "optimum" value may be unknown even after the problem is solved (see discussion in next section).

- If there is no convection i.e., $\boldsymbol{M}=(0,0)$, it follows from (62), (63) that

$$
k_{0 x}^{\mathrm{eff}}=k_{0 x}, \quad k_{0 y}^{\mathrm{eff}}=k_{0 y},
$$


which inserted in (61) yields

$$
\begin{aligned}
\tau_{\mathrm{SGS}}= & \frac{A_{\text {num }}}{A_{\text {den }}} \\
= & -\frac{1}{k_{0}^{2}}+\frac{6}{k_{0}^{4} h^{2}} \\
& \times \frac{\left[4-\cos \left(k_{0 x}^{\text {eff }} h\right)-\cos \left(k_{0 y}^{\text {eff }} h\right)-2 \cos \left(k_{0 x}^{\text {eff }} h\right) \cos \left(k_{0 y}^{\text {eff }}\right)\right]}{\left[2+\cos \left(k_{0 x}^{\text {eff }} h\right)\right]\left[2+\cos \left(k_{0 y}^{\text {eff }} h\right)\right]} .
\end{aligned}
$$

As expected, (65) is nothing but minus the stabilization parameter found from the GLS stabilization of the Helmholtz equation in [46].

- The one-dimensional counterpart of (61) can be obtained e.g., by setting $M_{y}=0(\varphi=0)$ and $k_{0 y}=0\left(\theta=0^{\circ}\right)$ in it and by taking into account that the effective wavenumber components (62) and (63) will become

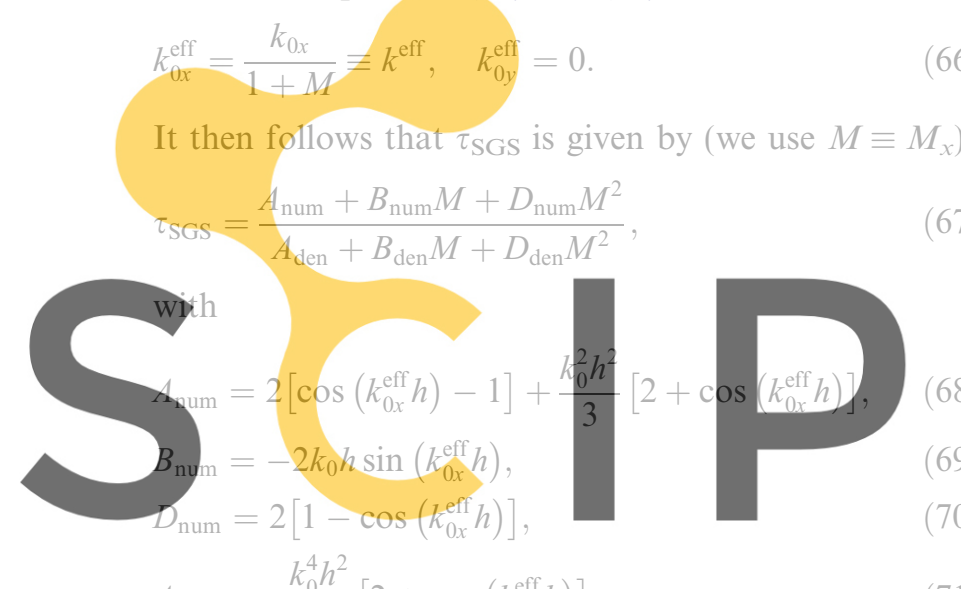

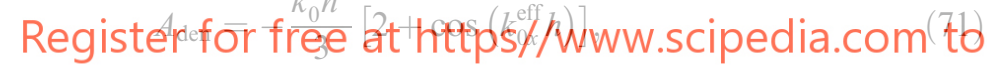

$$
\begin{aligned}
& B_{\text {den }}=0, \\
& D_{\text {den }}=4 k_{0}^{2} D_{\text {num }} .
\end{aligned}
$$

- The non-convective one-dimensional case follows from setting $M=0$ in (67) and noting that now (66) yields $k_{0}^{\text {eff }}=k_{0}$. The stabilization parameter then becomes

$\tau_{\mathrm{SGS}}=\frac{A_{\mathrm{num}}}{A_{\mathrm{den}}}=-\frac{1}{k_{0}^{2}}+\frac{6}{k_{0}^{4} h^{2}} \frac{\left[1-\cos \left(k_{0} h\right)\right]}{\left[2+\cos \left(k_{0} h\right)\right]}$,

which is nothing but minus the $\tau_{\mathrm{GLS}}$ obtained for the one-dimensional Helmholtz equation in [21,22].

- In [5] a general stabilization parameter for the Convection-Diffusion-Reaction equation was proposed. Also in [25] it was proposed to extend the stabilization parameter in [14] to account for the whole range of source terms (positive and negative). Although, as previously mentioned, the convected Helmholtz equation constitutes a particular case of the more general Convection-Diffusion-Reaction equation, it has some important particularities such as having a pure imaginary complex advection velocity. Care has then to be taken in the design of stabilization parameters for it. For instance, it is clear that the application of the above cited general stabilization parameters to the convected
Helmholtz equation cannot provide the right amount of stabilization. This is so because these parameters only depend on the modulus of the advection velocity but not on its direction, whereas it is clear from Fig. 4a and numerical experiments that very different stabilization values are required e.g., for upstream or downstream propagating waves.

In Fig. $4 \mathrm{a}$ we have plotted the dependence of $-\tau_{\text {SGS }}$ with $k_{0} h$ for various Mach numbers in the one-dimensional case, see (67). We can observe the role played by the Doppler effect: for positive Mach numbers (downstream propagation) the effective wavenumber $k_{0}^{\text {eff }}$ is smaller than $k_{0}$ (see (66)) so that less stabilization is required and $\left|\tau_{\mathrm{SGS}}\right|$ is smaller than for the Helmholtz case $(M=0$, Eq. (74)). On the opposite, for negative Mach numbers (upstream propagation) the effective wavenumber is larger than $k_{0}$ and consequently more stabilization is required than for the Helmholtz case.

In Fig. $4 \mathrm{~b}$ we have plotted the dependence of $-\tau_{\mathrm{SGS}}$ with $k_{0} h$ for a plane wave having a wavenumber vector in polar coordinates $\boldsymbol{k}_{0}=\left(k_{0}, \theta\right)=\left(24,50^{\circ}\right)$ propagating in uniform flows characterized by Mach number vectors $M=(M, \varphi)$,

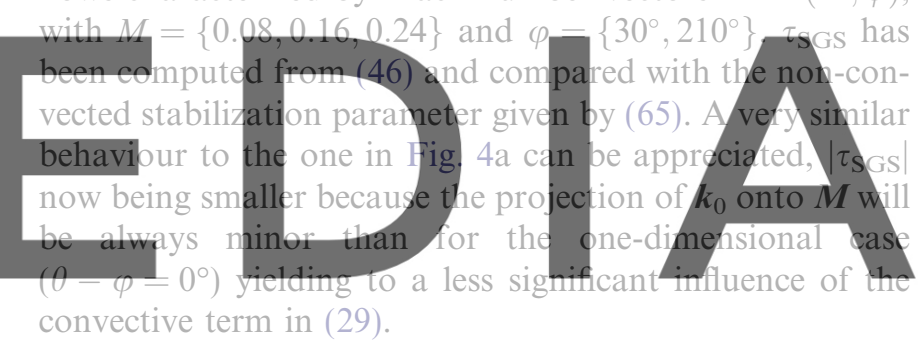

convective term in (29).

ownload the version without the watermark 3.5. Discussion

- It is clear that due to the Doppler effect different mesh resolutions should be used to properly capture an acoustic field containing upstream and downstream propagating waves in a uniform mean flow. In [15] it was shown that even if we were able to build a mesh keeping the number of points per wavelength constant (hence avoiding the lack of resolution due to the Doppler effect), the pollution effects for the Galerkin finite element solution of the convected wave equation still differ for upstream and downstream propagation waves. It was also shown that a large degree of anisotropy is detected when analyzing the dispersion and amplitude error dependencies on wave and flow orientations [16]. As a conclusion, it was suggested that the use of mesh adaptative strategies based, for instance, on a posteriori error estimations, may be useful to deal with complex acoustic fields with waves propagating in many directions. Obviously, this can be a good procedure although it requires either the performance of several simulations (at least two) or a previous knowledge of the resulting acoustic field to build an adequate mesh. However, the former might turn rather unpractical for large problems while the latter rarely occurs. An alternative (or complement) to the 
adaptative mesh strategy is the use of stabilization techniques. If we were able to compute the right amount of stabilization needed at each mesh zone to yield a good solution, it would not be necessary to modify the mesh. In the previous sections we have presented a finite element method to do so.

- As pointed out in the first remark of Section 3.4, while the flow orientation $\varphi$ is a given quantity, the wavenumber direction $\theta$ will be unknown prior to the solution of the problem. The resulting $\tau_{\mathrm{SGS}}$ will consequently depend on the values chosen for $\theta$ and the stabilization effect may be significant for waves propagating in this direction but much weaker or inexistent for other ones. As already mentioned in the introduction, this is probably the main drawback of obtaining the stabilization parameter from a dispersion analysis in the ASGS (or GLS) stabilization for the Helmholtz equation.

A considerable amount of literature exists with numerical simulations confirming that the GLS finite element method with $\theta=0^{\circ}, 22.5^{\circ}$ performs better than the Galerkin method in a large variety of cases, not only for the wave angles indicated. This is demonstrated for example in [38], where it is shown that for a particular
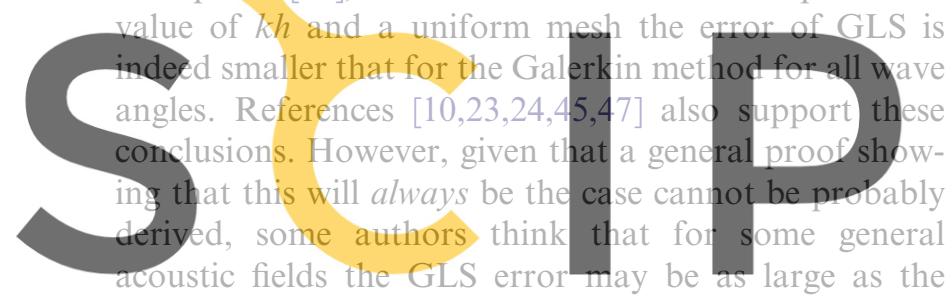

Galerkin one. For example, in [29, p. 164], it is claimed

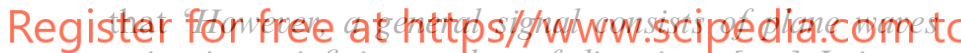
going in an infinite number of directions [...] It is not clear if the GLS leads to improved approximation of a wave that is not dominant in the preferred direction. In fact, numerical tests [...] show that the GLS-FEM has the same error as the standard Galerkin FEM if the direction of the exact solution is different from the direction chosen for the factor $\tau$ " and the author refers to [46]. Nevertheless, our numerical experiments with the convected Helmholtz equation, some of which are shown below, seem to confirm that results are always better using the stabilized finite element formulation, in spite of the fact that the stabilization parameter is computed for a given wave angle ( $0^{\circ}$ in our calculations). However, we do not intent to draw any general conclusion out of this result, particularly considering the controversy mentioned above. In any case, it could be possible that at some particular points the error may be locally higher than the Galerkin one.

\section{Numerical examples}

\subsection{Plane wave in a uniform mean flow}

As a first numerical example we consider the case of finding the acoustic pressure in a computational domain,
$\Omega_{\mathrm{ac}}=(0,1) \times(0,1)$, with inhomogeneous Dirichlet boundary conditions on $\partial \Omega_{\text {ac }}$ and normal surface vector $\boldsymbol{n}$ pointing outwards of the domain (see Fig. 3), such that

$\left[\nabla^{2}+\left(k_{0}+\mathrm{i} \boldsymbol{M} \cdot \nabla\right)^{2}\right] \hat{p}=0 \quad$ in $\Omega_{\mathrm{ac}}$,

$\hat{p}=\exp \left[\mathrm{i}\left(k_{0 x}^{\mathrm{eff}} x+k_{0 y}^{\mathrm{eff}} y\right)\right] \quad$ on $\partial \Omega_{\mathrm{ac}}$.

For the Mach number vector characterizing the mean flow we take the values in polar coordinates $\boldsymbol{M}=(M, \varphi)=$ $\left(0.6,30^{\circ}\right)$ and for the wavenumber vector we use $\boldsymbol{k}_{0}=$ $\left(k_{0}, \theta\right)=\left(24 \mathrm{~m}^{-1}, 50^{\circ}\right)$ to be inserted in (75), (76). The effective wavenumber $\boldsymbol{k}_{0}^{\text {eff }}=\left(k_{0 x}^{\text {eff }}, k_{0 y}^{\text {eff }}\right)$ in (76) is computed from (62), (63).

The exact solution for the problem above is the plane wave

$\hat{p}=\exp \left(i k_{0}^{\mathrm{eff}} \cdot x\right)$

propagating with an effective wavenumber in polar coordinates $\boldsymbol{k}_{0}^{\mathrm{eff}}=\left(k_{0}^{\mathrm{eff}}, \theta^{\mathrm{eff}}\right)=\left(16.35 \mathrm{~m}^{-1}, 68.8^{\circ}\right)$. Hence, the effect of the mean flow on the original wave, having wavenumber $\boldsymbol{k}_{0}$, is to reduce the modulus of the wavenumber and to change its direction upstream.

If we now proceed to solve (75), (76) using the Galerkin finite element method (31) on a uniform grid with elements
of size $h \times h$, with $h=0.025$, we will observe that even
though $k_{0}^{\text {eff }} h=0.4$ (more than the recommended ten points
per wavelength), the method is unable to yield an accurate
solution for the problem. On the contrary, the stabilized
ASGS finite element approach using the modrfied weak
form (35) and the stabilization parameter (46) has been designed to yield exact nodal values for this case. This

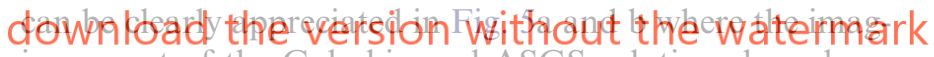
inary part of the Galerkin and ASGS solutions have been respectively plotted. It is apparent from Fig. 5a that the Galerkin solution distorts the shape of the wave and yields much higher amplitudes than the correct ones. This can also be observed in Fig. 6, where the imaginary part of the acoustic pressure in a one-dimensional cut of the domain, $\left.\Omega_{\mathrm{ac}}\right|_{x=0.25}:=\{(0.25, y) \mid 0<y<1\}$ is plotted for both cases, together with the exact solution.

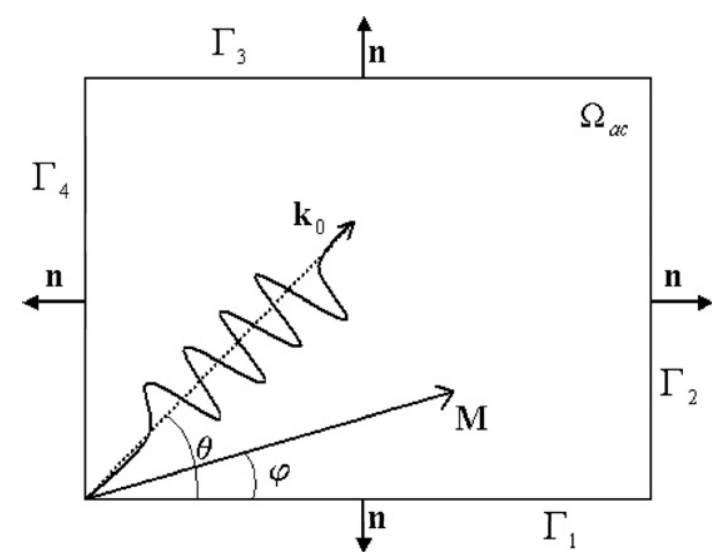

Fig. 3. Scheme for a plane wave propagating with $\boldsymbol{k}_{0}$ in a uniform flow characterized by $\boldsymbol{M}$. 


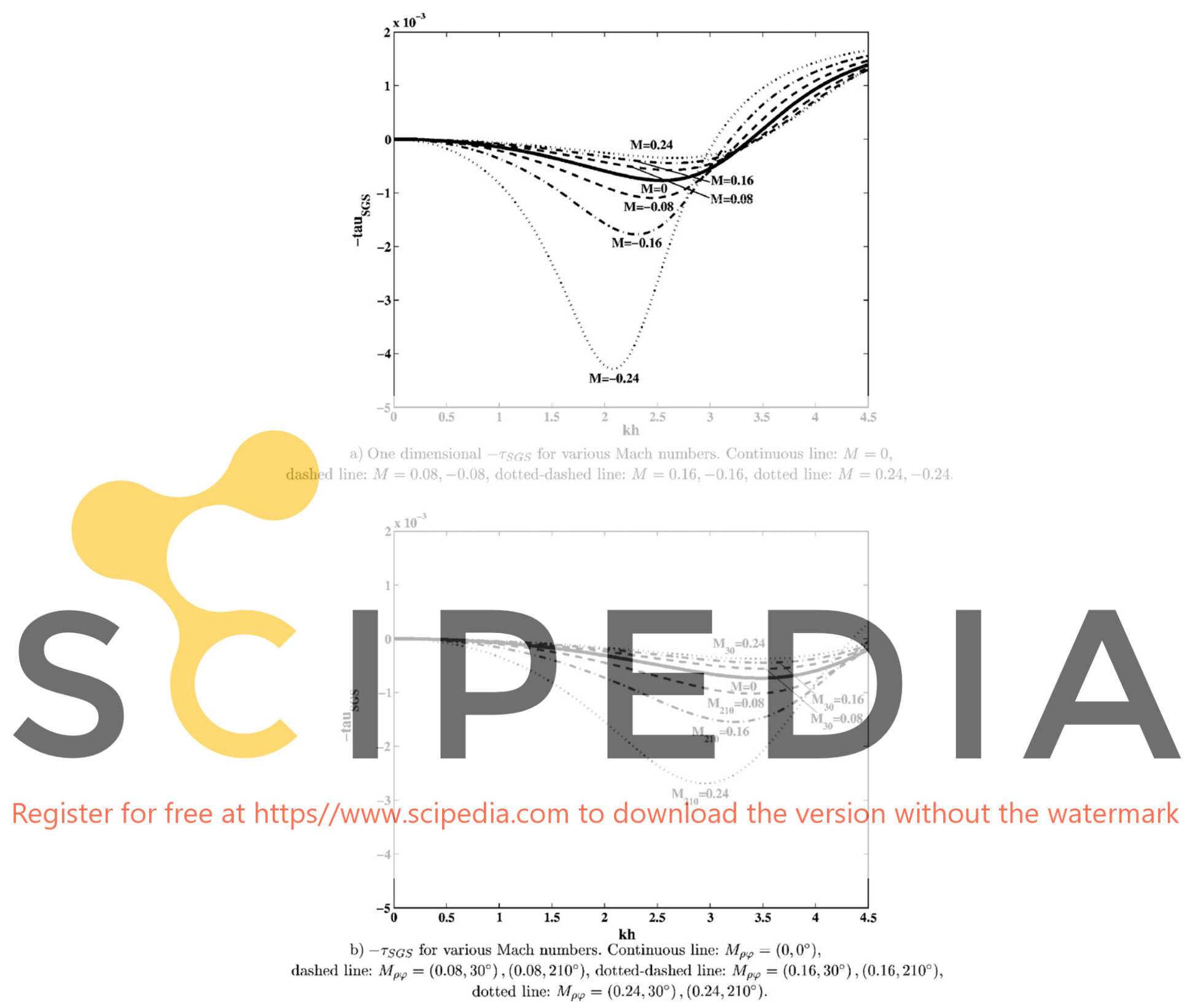

Fig. 4. Stabilization parameter dependence with Mach number.

On the other hand, to make the stabilized ASGS method useful in as many situations as possible, it should perform well for other meshes than the structured bilinear quadrilateral elements for which the stabilization parameter $\tau_{\mathrm{SGS}}$ has been optimized. To check this point we have constructed an unstructured mesh of quadrilaterals elements (see Fig. 7) and studied the dependence of the numerical solution relative error in the $L^{2}$-norm, $\left\|\hat{p}_{h}-\hat{p}_{\text {exact }}\right\| /$ $\left\|\hat{p}_{\text {exact }}\right\|$, when refining the mesh. $\hat{p}_{\text {exact }}$ is given by (77) and $\hat{p}_{h}$ denotes the numerical solution obtained by either the Galerkin method or by the stabilized ASGS one. Results are plotted in Fig. 8a together with the -2 slope of the best approximation solution. It can be clearly observed that the ASGS stabilized solution improves the results of the
Galerkin method for all meshes. Moreover, we have plotted the results of using the ASGS formulation with the stabilization parameter, $\tau_{\mathrm{SGS}}$, corresponding to $\theta=0^{\circ}$ instead of $\theta=50^{\circ}$. It can be seen that the solution is also better than the Galerkin one (and for some meshes even better than the $\theta=50^{\circ}$ one). However, this cannot be taken as a general result stating that any value of $\theta$ in $\tau_{\text {ASGS }}$ would result in an improvement of the solution (see the discussion in Section 3.5).

Further tests are presented in Fig. 8b and c. In Fig. 8b we have considered the same Mach number and meshes than for Fig. 8a, but for a plane wave with wavenumber $\boldsymbol{k}_{0}=\left(24 \mathrm{~m}^{-1}, 80^{\circ}\right)$. This results in a free plane wave with effective wavenumber $\boldsymbol{k}_{0}^{\text {eff }}=\left(23 \mathrm{~m}^{-1}, 116^{\circ}\right)$, i.e., almost 


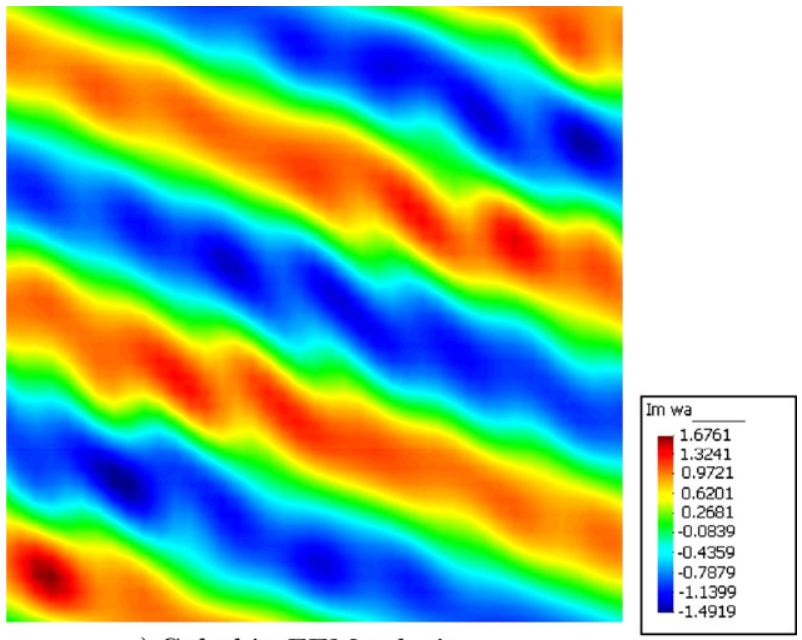

a) Galerkin FEM solution

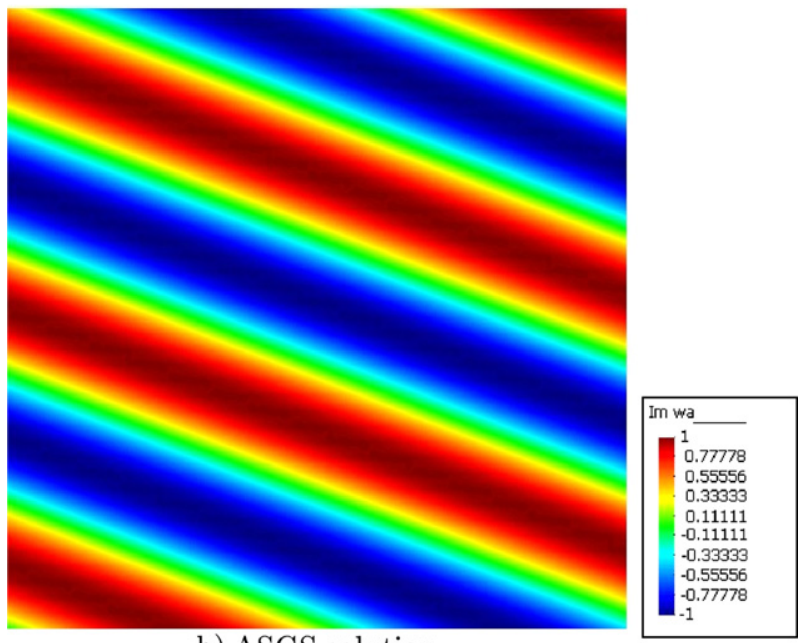

b) ASGS solution

Fig. 5. Imaginary part of the Galerkin and ASGS finite element solutions to problem (75), (76) in text.

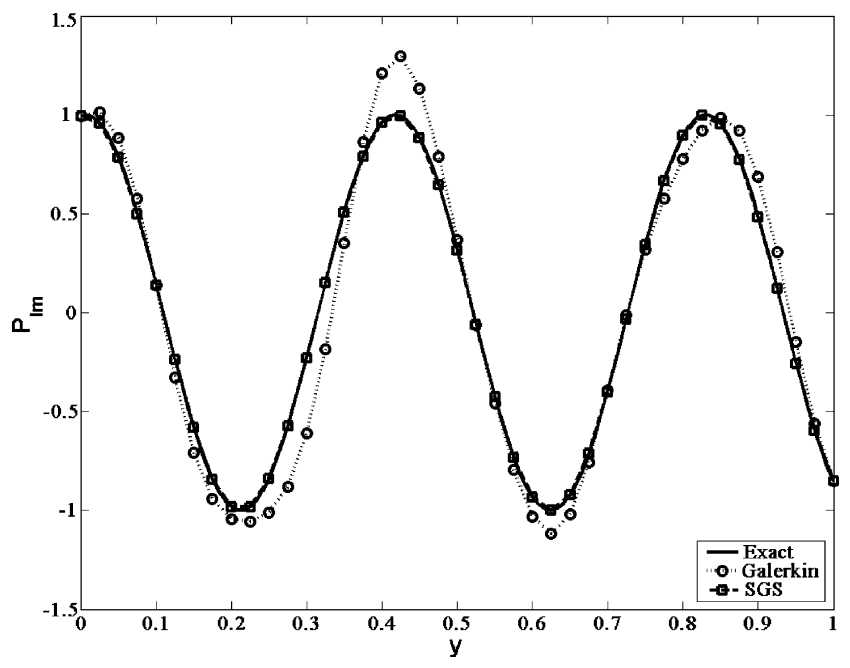

Fig. 6. Imaginary part of the reference, Galerkin and SGS solutions for a one-dimensional cut of the domain corresponding to the Dirichlet problem (75), (76) in text.

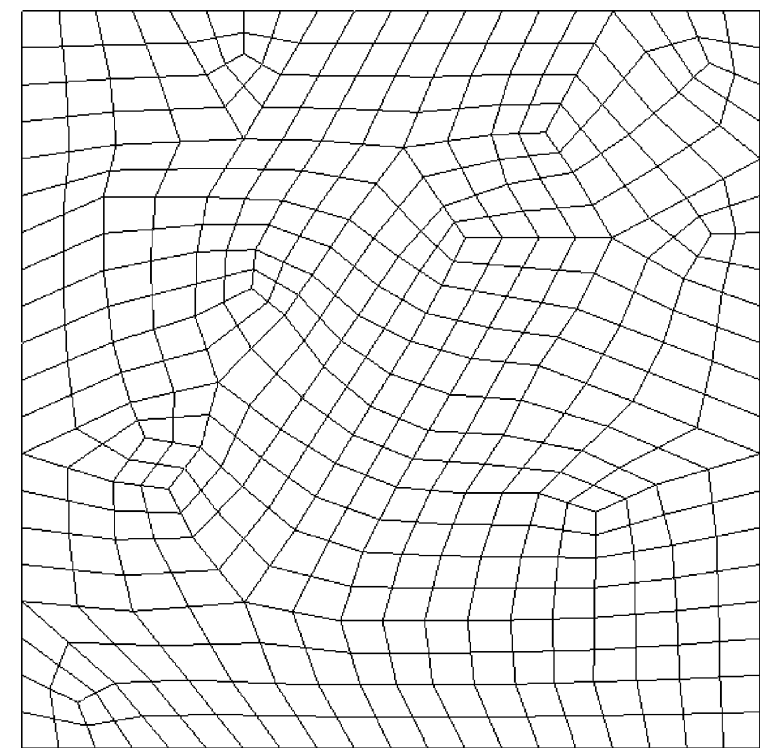

Fig. 7. Unstructred mesh of quadrilateral elements.

propagating in the normal direction to the mean flow. It can be observed in In Fig. $8 \mathrm{~b}$ that once the resolution threshold is surpassed $\left(h^{-1} \gtrsim 50\right)$ the ASGS solution error is clearly lower than the Galerkin one. The ASGS solution taking $\theta=0^{\circ}$ in the stabilization parameter also yields better solutions. On the opposite, if using the ASGS stabilization without taking into account convection (65), large errors are encountered depending on the mesh, either when setting $\theta=0^{\circ}$ or $\theta=80^{\circ}$ in the stabilization parameter.

Next we have repeated the test but for a plane wave having wavenumber $\boldsymbol{k}_{0}=\left(24 \mathrm{~m}^{-1}, 190^{\circ}\right)$. Given that this will result in a very large effective wavenumber modulus, we have reduced the speed of the uniform flow in order to achieve proper resolution using the same meshes of the previous examples. A flow with Mach number $\boldsymbol{M}=\left(0.2,30^{\circ}\right)$ has been considered. The resulting free wave has an effective wavenumber of $\boldsymbol{k}_{0}^{\text {eff }}=\left(29.7 \mathrm{~m}^{-1}, 194^{\circ}\right)$, which is traveling quite prone to the reverse direction of the mean flow, $210^{\circ}$. The numerical errors when using the various stabilization and Galerkin methods are plotted in Fig. 8c. In this case it can be also clearly observed how the ASGS method gives a better solution than the Galerkin one, once the necessary resolution threshold has been surpassed. It can also be seen that the ASGS method taking $\theta=0^{\circ}$, and the ASGS without considering convection for $\theta=0^{\circ}, 190^{\circ}$ improve now the Galerkin solution.

On the other hand, note the disparity of errors for fixed $h$ in Fig. $8 \mathrm{a}-\mathrm{c}$ ( $M=0.2$ for the latter) in accordance with the highly anisotropic error values found in [16], when analyzing the error dependence on the flow and wave orientations. In this line, note that the improvement of the solution when using stabilization in the analyzed cases strongly depends on the relative direction of the wave with respect to the mean flow. Better relative results have been obtained when comparing with the Galerkin solution for 


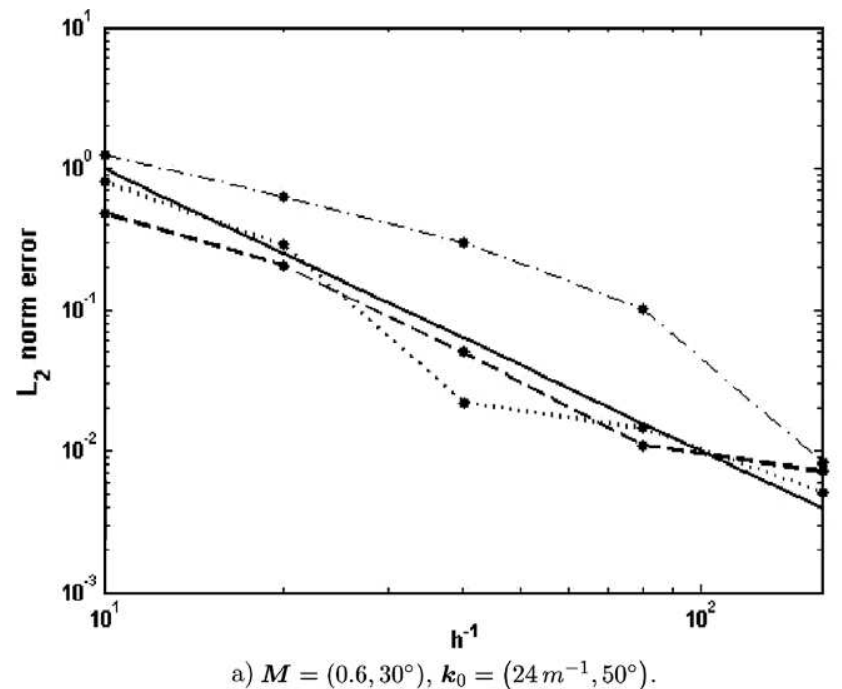

a) $\boldsymbol{M}=\left(0.6,30^{\circ}\right), \boldsymbol{k}_{0}=\left(24 \mathrm{~m}^{-1}, 50^{\circ}\right)$.

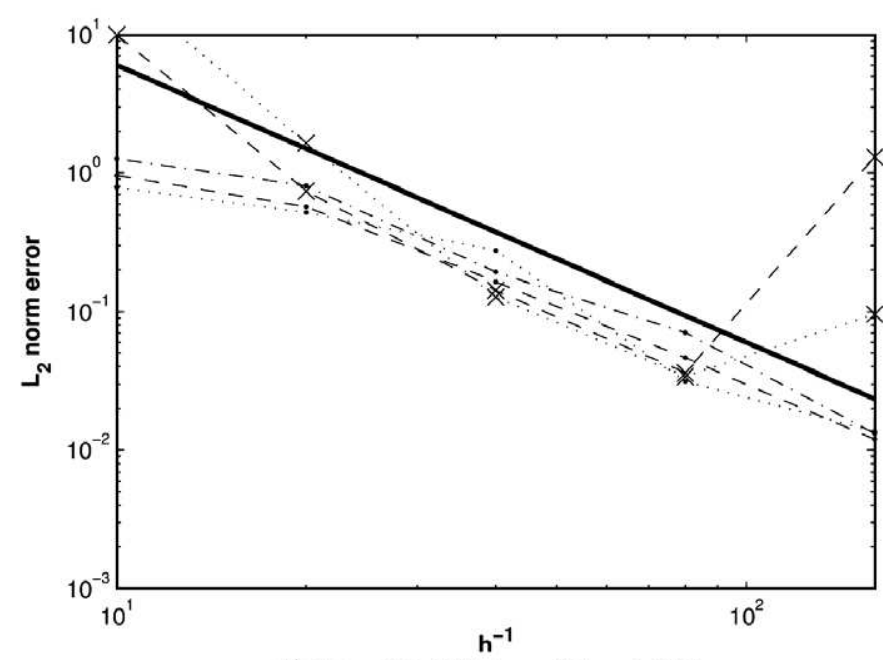

b) $M=\left(0.6,30^{\circ}\right), k_{0}=\left(24 m^{-1}, 80^{\circ}\right)$.

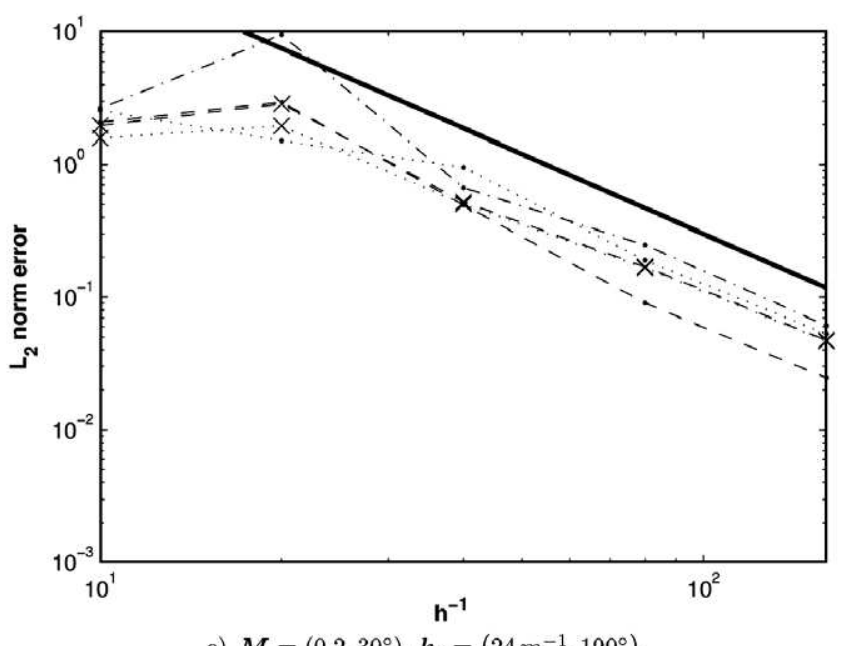

Fig. 8. Numerical solution error for propagating plane wave. (a) Dashed-dotted: Galerkin, dashed: ASGS with $\theta=50^{\circ}$, dotted: ASGS with $\theta=0^{\circ}$, Continuous line: -2 slope. (b) Dashed-dotted: Galerkin, dashed: ASGS with $\theta=50^{\circ}$, dotted: ASGS with $\theta=0^{\circ}$, cross-dashed: ASGS (M=0) and $\theta=50^{\circ}$, cross-dotted: ASGS $(\boldsymbol{M}=0)$ and $\theta=0^{\circ}$, continuous line: -2 slope. (c) Dashed-dotted: Galerkin, dashed: ASGS with $\theta=50^{\circ}$, dotted: ASGS with $\theta=0^{\circ}$, cross-dashed: ASGS $(\boldsymbol{M}=0)$ and $\theta=50^{\circ}$, cross-dotted: ASGS $(\boldsymbol{M}=0)$ and $\theta=0^{\circ}$, continuous line: -2 slope.

waves propagating close or reversal to the flow direction than for waves propagating normal to it.

\subsection{Aerodynamic sound radiated by flow past a cylinder}

As a second numerical example, we will apply the methodology presented in the previous sections to the aerodynamic acoustic field generated by flow past a twodimensional circular cylinder. The purpose of this example will be to check the performance of the stabilized formulation in a somehow more intricate problem than the usual benchmark tests. We will check if the stabilization formulation derived in Section 3 is able to yield good results for this case that contains waves propagating in several directions that have been computed in an unstructured mesh of linear triangular elements (again a different mesh than the one used to optimize $\tau_{\mathrm{SGS}}$ for a single propagation direction).

\subsubsection{The physical problem: Aeolian tones}

Let us consider a two-dimensional cylinder with diameter $D$ embedded in a flow with uniform free stream velocity in Cartesian coordinates $\boldsymbol{U}_{0}=\left(U_{0}, 0\right)$ and Mach number $\boldsymbol{M}=(M, 0)=\left(U_{0} / c_{0}, 0\right)$. The Reynolds number based on these variables is given by $R e=\rho_{0} U_{0} D / \mu$, with $\mu$ being the dynamic coefficient of viscosity. The flow dynamics behind the cylinder strongly depends on the Reynolds number value. When increasing Re from an almost zero value to larger and larger values of $R e$, a set of bifurcations take place that bring the flow from a steady and totally symmetric configuration to a fully developed turbulent flow, probably following the Ruelle-Takens-Newhouse route to turbulence (see e.g., [26,11]).

We will focus here on the problem once the flow first loses its steadiness and a wake of alternating periodic vortices is formed behind the cylinder. The set of these shed vortices is known as the von Kármán vortex street. Vortex 
shedding induces lift fluctuations on the cylinder (drag fluctuations also occur although they are much smaller, see $[42,18]$ c.f. $[26,31])$, which lead to the radiation of sound, having a dipole pattern. Remember that the frequency of the radiated sound is the same as the vortex shedding frequency and is given by

$f_{\text {vsh }}=S_{t} U_{0} / D$

where $S_{t}$ is the Strouhal number that has the Reynolds number dependency ([42] c.f. [26])

$S_{t}=0.198(1-19.7 / R e), \quad R e<5 \times 10^{5}$.

The radiated sound is known as an aeolian tone. In practical cases the cylinder can often vibrate as a reaction to the changes in lift. This motion tends to correlate the vortex shedding along the length of the cylinder (three-dimensional case) resulting in a high level of radiated noise. If in addition, the frequency of the vortex shedding coincides with a natural mode of vibration of the cylinder, the mechanism is further reinforced. This mechanism is responsible, for instance, of wire whistles when wind is blowing and is of importance in some industrial problems such as noise generated by the pantographs of high-speed trains or noise generation from heat exchangers.

In order to compute the aeroacoustic source term we have made use of Lighthill's acoustic analogy [34]. Lighthill reordered the continuity and momentum equations for a compressible flow to obtain an inhomogeneous wave equation for the density fluctuations given by

$\left(\partial_{t t}^{2}-c_{0}^{2} \nabla^{2}\right)\left(\rho^{\prime}\right)=(\nabla \otimes \nabla): \boldsymbol{T}$.

In $(80) \rho^{\prime}:=\rho-\rho_{0}$ stands for the density fluctuations and $\boldsymbol{T}$ is known as the Lighthill tensor, that has the expression

$\boldsymbol{T}:=\rho(\boldsymbol{u} \otimes \boldsymbol{u})+\left(p^{\prime}-c_{0}^{2} \rho^{\prime}\right) \boldsymbol{I}-\boldsymbol{\sigma}$.

with $\boldsymbol{u}$ standing for the velocity vector, $p^{\prime}:=p-p_{0}$ for the pressure fluctuations and $\sigma$ for the Cauchy stress tensor.
However, nothing is gained from (80), (81) unless $\boldsymbol{T}$ is approximated somehow, because these equations retain the full complexity of solving the compressible NavierStokes equations. Consequently, the powerfulness of Lighthill's approach precisely relies in finding an appropriate approximation for the tensor $\boldsymbol{T}$, therefore simplifying the original problem. For the case of incompressible flows $(M \leqslant 0.3)$, Lighthill himself proposed to use the Reynolds stress tensor

$\boldsymbol{T}:=\rho_{0}(\boldsymbol{u} \otimes \boldsymbol{u})$,

where $\boldsymbol{u}$ is now the velocity resulting from the solution of the incompressible Navier-Stokes equations (i.e., we take $\nabla \cdot \boldsymbol{u}=0$ ). The validity of such an approach was deeply analyzed in [9] by the method of Matched Asymptotic Expansions and more recently, e.g., in [43,44].

To compute the aeroacoustic field a three-step process has been carried out, which is similar to the one in $[40,41]$. This process will be only briefly outlined here and it will be the subject of a forthcoming paper (some details can be found for the moment in [19]). The first step of the method consists of a time-dependent fluid dynamic computation (CFD) of the incompressible flow impinging on the cylinder that has served to obtain the solenoidal velocity field on the computational domain. The incompressible Navier-Stokes equations have been solved by means of an algebraic SGS method (see e.g., [27,5-7]) to circumvent the fulfilment of the inf-sup condition when using equal interpolation for the velocity and pressure fields, and to avoid the instabilities arising in convection dominated flows. We have used the stabilization parameters in [5] (see also [6] for a different derivation of these parameters).

The second step of the method consists in computing the aeroacoustic source term in (80) from the velocity field of the first step and using the Reynolds stress tensor approximation (82). As this source term contains a double

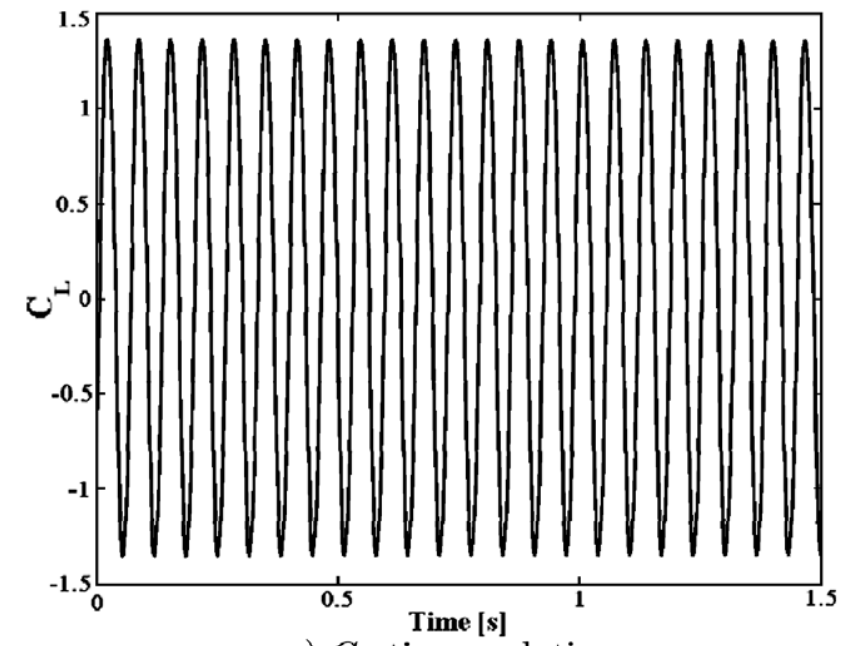

a) $C_{L}$ time evolution.

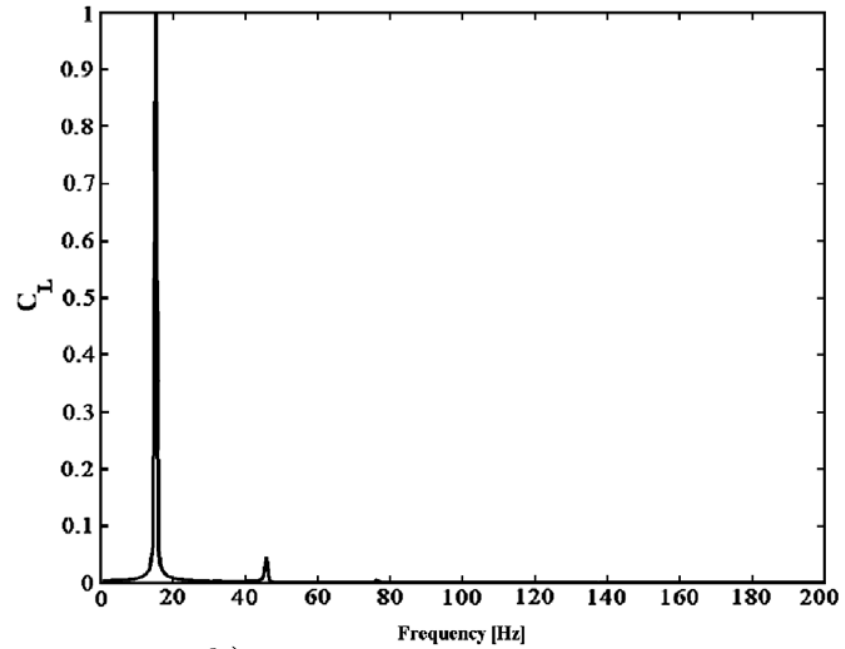

b) $C_{L}$ normalized spectrum.

Fig. 9. Temporal evolution and spectrum of the cylinder lift coefficient, $C_{L}$. 
divergence, we have made use of the following approximation in order to use $C^{0}$-continuous finite elements in the computation:

$$
\begin{aligned}
(\nabla \otimes \nabla) & : \boldsymbol{T} \sim \rho_{0}(\nabla \otimes \nabla):(\boldsymbol{u} \otimes \boldsymbol{u}) \sim \rho_{0}(\nabla \otimes \boldsymbol{u}) \\
& :(\nabla \otimes \boldsymbol{u})^{\mathrm{T}}=: s_{L}(\boldsymbol{x}, t),
\end{aligned}
$$

where the incompressibility constraint $\nabla \cdot \boldsymbol{u}=0$ has been applied twice in the second equality of (83) and we have denoted this approximated source term by $s_{L}(\boldsymbol{x}, t)$. Once (83) is computed, we have Fourier transformed it to obtain its frequency domain counterpart.

The third step of the method finally consists in computing the acoustic field generated by the uniform flow of speed $\boldsymbol{U}_{0}=\left(U_{0}, 0\right)$ impinging on the cylinder. We have considered two cases: the first one corresponds to the straightforward application of Lighthill's acoustic analogy. This has given the acoustic field as seen by an observer that is at rest with the cylinder and feels the uniform inflow impinging on it at speed $\left(U_{0}, 0\right)$. Actually, we have been interested in knowing the acoustic field spatial distribution at the vortex shedding frequency, which is given by the time Fourier transform of Lighthill's equation

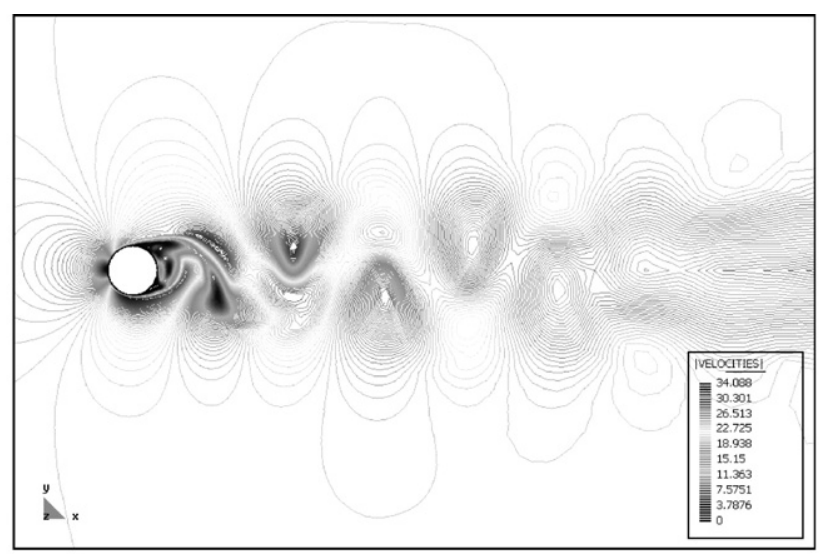

a) Isovelocity contourlines showing von Kármán vortex street.

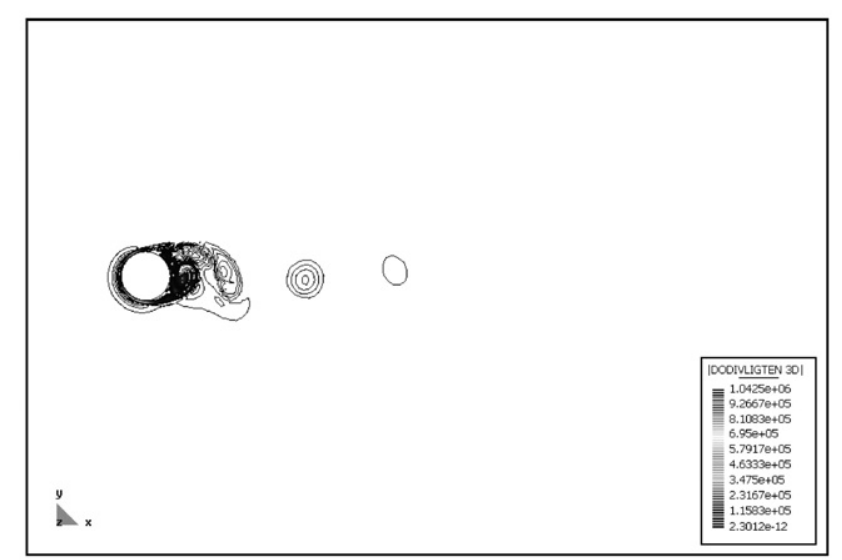

b) Snapshot of the acoustic source term (Lighthill's tensor double divergence).

Fig. 10. CFD results. $-\left(\nabla^{2}+k_{0}^{2}\right) \hat{p}=\hat{s}_{L} \quad$ in $\Omega_{\mathrm{ac}}$,

$\nabla \hat{p} \cdot \boldsymbol{n}=0 \quad$ on $\Gamma_{\text {cyl }}$,

$\nabla \hat{p} \cdot \boldsymbol{n}=\mathrm{i} k_{0} \hat{p} \quad$ on $\Gamma_{\infty}$,

with $k_{0}=\omega / c_{0}=2 \pi f_{\mathrm{vsh}} / c_{0}, \quad \Omega_{\mathrm{ac}} \subset \mathbb{R}^{2}$ being a bounded domain, $\Gamma_{\text {cyl }}$ the cylinder boundary and $\Gamma_{\infty}$ the far field boundary of $\Omega_{\mathrm{ac}}$.

The second case has consisted in considering the acoustic field as seen by an observer at rest with the flow that sees the cylinder approaching at speed $\left(-U_{0}, 0\right)$. To obtain this result we can perform a full Lorentz transformation of Lighthill's Eq. (84) (note that the source term is Galilean invariant so it will not be affected by the uniform mean flow). This results in the convected Helmholtz equation:

$\left[\nabla^{2}+\left(k_{0}+\mathrm{i} \boldsymbol{M} \cdot \nabla\right)^{2}\right] \hat{p}=\hat{s}_{L} \quad$ in $\Omega_{\mathrm{ac}}$,

$\nabla \hat{p} \cdot \boldsymbol{n}=-\mathrm{i} k_{0} \alpha_{N} \hat{p} \quad$ on $\Gamma_{\text {cyl }}$,

$\nabla \hat{p} \cdot \boldsymbol{n}=\mathrm{i} k_{0} \alpha_{S} \hat{p} \quad$ on $\Gamma_{\infty}$.

Notice that the low Mach number limitation for this example does not arise from the convected wave equation, which is valid up to transonic flows, but from the use of the Reynolds tensor as an approximation for Lighthill's tensor, which is only valid for incompressible flows $(M<0.3)$. Note also that the convected Helmholtz equation (87)(89) only applies to uniform flows, which is not the case for the vortex street past the cylinder. However, Lighthill's acoustic analogy establishes a clear separation between the acoustic source zone, where acoustic waves are generated (in this case the boundary of the cylinder and the periodic vortices past it) and the propagation zone (acoustic medium) where acoustic waves are radiated and propagate (in this case the remaining of the domain). Hence one can imagine the problem as that of a set of acoustic sources placed near the cylinder (with independence of the fact that they have been obtained from a CFD computation of a non-uniform flow) radiating into an acoustic medium that may be at rest or moving e.g., at uniform speed. The convected Helmholtz equation can be applied to this acoustic medium.

\subsubsection{Numerical results}

For the numerical example we have considered a circular cylinder of diameter $D=0.3$ in a circular computational domain $\Omega_{\mathrm{CFD}}$ of diameter $3 \times 10^{3} \mathrm{D}$. We have taken a dynamic viscosity coefficient of $\mu=0.006$ and an impinging flow velocity in Cartesian coordinates of $\boldsymbol{U}_{0}=(20,0)$, leading to Reynolds and Mach numbers at the far field (away from the cylinder) of $R e=10^{3}$ and $\boldsymbol{M}=(5.83 \times$ $10^{-2}, 0$ ), for a sound speed of $c_{0}=343$ (all units are in SI). The incompressible Navier-Stokes problem has been solved in an unstructured mesh of triangular elements ranging in size from $\sim 3 \times 10^{-3} D$ near the cylinder surface to $\sim 30 D$ at the far field.

In what concerns the acoustic field, computations have been performed in the same domain used for the CFD computation, i.e., $\Omega_{\mathrm{ac}}=\Omega_{\mathrm{CFD}}$. As there is no analytical 


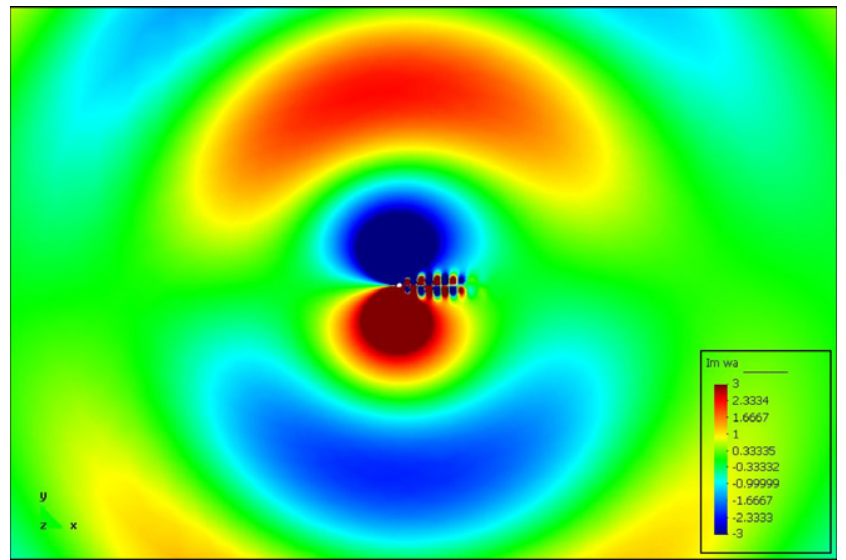

Fig. 11. Near field results for the imaginary part of the convected reference acoustic pressure.

solution for this problem, the Galerkin finite element method has been first used to solve Eqs. (84)-(89) in a very

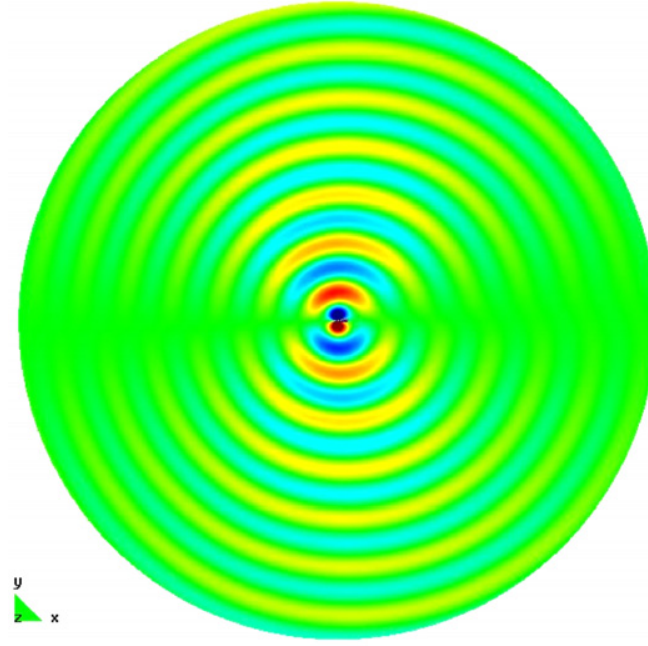

a) Reference convected Helmholtz

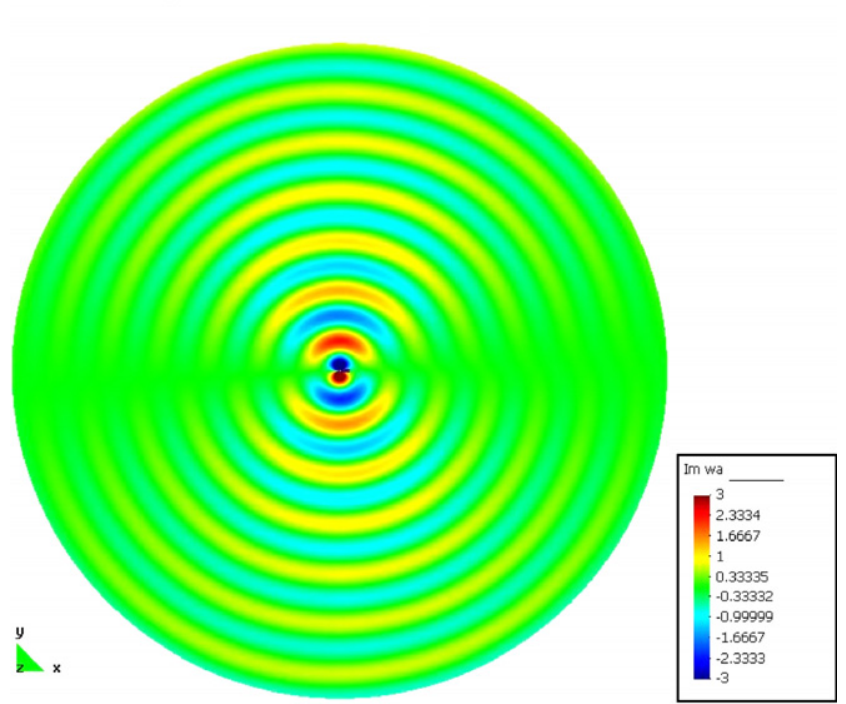

b) Reference Helmholtz

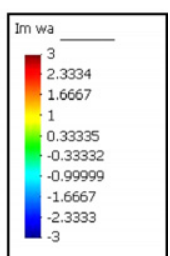

Fig. 12. Reference far field results for the imaginary part of the acoustic pressure.

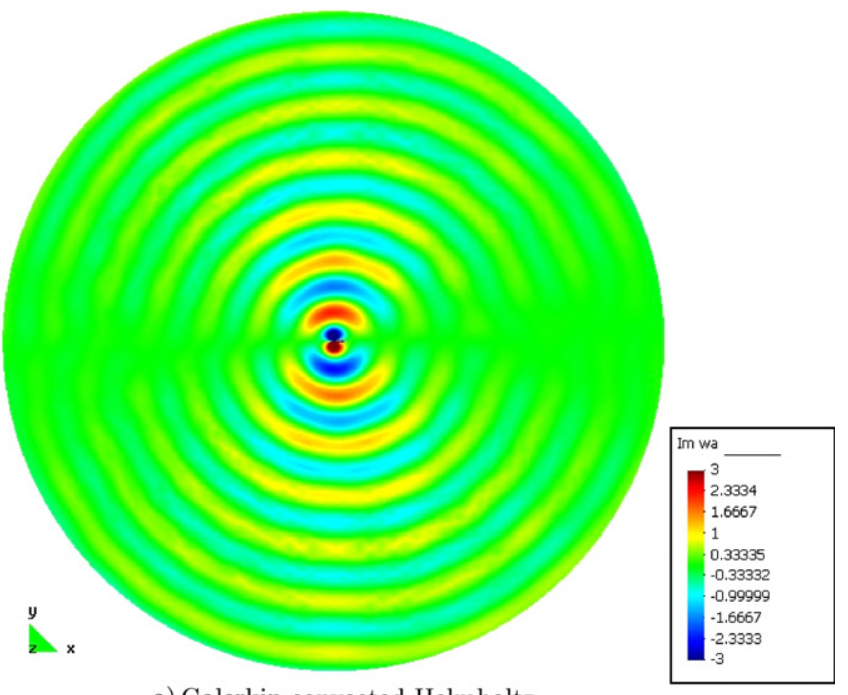

a) Galerkin convected Helmholtz

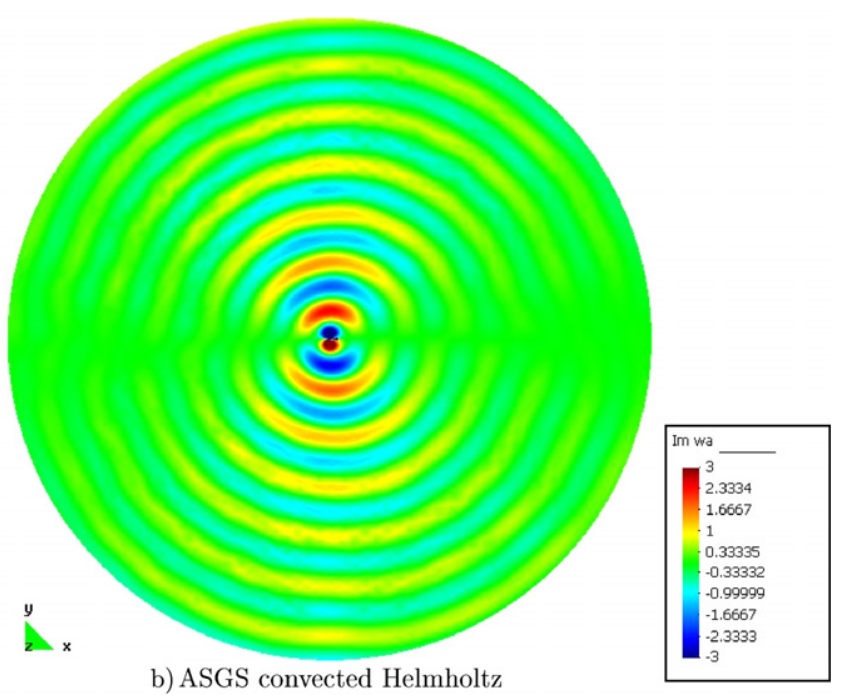

Fig. 13. FEM far field results for the imaginary part of the acoustic pressure.

fine mesh that has a high resolution at the far field. It has been checked that the obtained results show no appreciable differences with the results obtained using the SGS stabilization in the same mesh. The solution corresponding to this fine mesh will be termed hereafter as reference solution for the simplicity of notation and used to assess the performance of the method in the line of what is done in [23]. In order to verify the performance of the implemented ASGS stabilization for the convected Helmholtz equation, problem (87)-(89) has been also solved in a rather coarse mesh that has a resolution of 7-8 nodes per wavelength at the far field. The Galerkin finite element method and the ASGS stabilized finite element method have been used to solve (87)-(89) in the coarse mesh and their solutions have been compared with the reference solution to see which one performs better.

Let us first have a look at the results from the CFD computation. A periodic flow is established with vortex shedding at a frequency of $f_{\mathrm{vsh}}=15.3 \mathrm{~Hz}\left(S_{t}=0.229\right)$. This 
can be appreciated in Fig. 9a and b, where the temporal evolution and normalized spectrum of the lift coefficient are plotted. The lift coefficient has a mean amplitude of $\sim 1.36$. The computed frequency and Strouhal number are slightly higher than the ones obtained from (78), (79), $f_{\mathrm{vsh}}=13 \mathrm{~Hz}\left(S_{t}=0.194\right)$. This is in part due to the fact that (78), (79) are valid for three-dimensional cylinders, while we are performing two-dimensional simulations. The three-dimensional effects begin to be significant for $R e>300$ and two-dimensional simulations tend to over predict the values for $f_{\text {vsh }}$ and $S_{t}$ (see [36] and references therein).

In Fig. 10a we have plotted a snapshot of the isovelocity contourlines at a given instant of time showing the von Kármán vortex street. In Fig. 10b we present the contour- lines corresponding to the acoustic source term $s_{L}=$ $\rho_{0}(\nabla \otimes \boldsymbol{u}):(\nabla \otimes \boldsymbol{u})^{\mathrm{T}}$ used as an approximation for Lighthill's tensor. It can be observed that the source term rapidly decreases to zero when moving away from the cylinder surroundings. The fast decay of the source term is of crucial importance and in fact justifies the acoustic analogy approaches. Otherwise, it would not be possible to distinguish between a source region and a propagating one (see e.g., $[9,8])$.

In what concerns the acoustic results, the imaginary part of the reference acoustic pressure for the convected Helmholtz Eqs. (87)-(89) in the near field is shown in Fig. 11. It can be clearly observed that although there is sound generated at the wake of the cylinder, only sound having a dipole pattern and generated by lift fluctuations on the

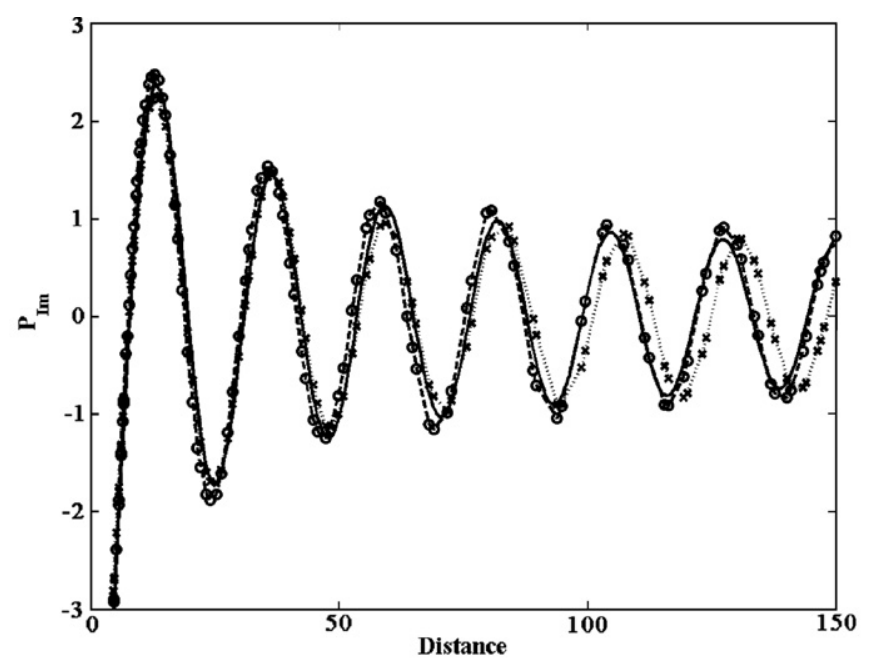

a) Imaginary part of the reference, Galerkin and SGS solutions

for a one dimensional cut of the domain at $90^{\circ}$ with respect to the $\mathrm{x}$-axis.

Continuous line: reference solution, Dashed with circles: ASGS, Dotted cross: Galerkin.

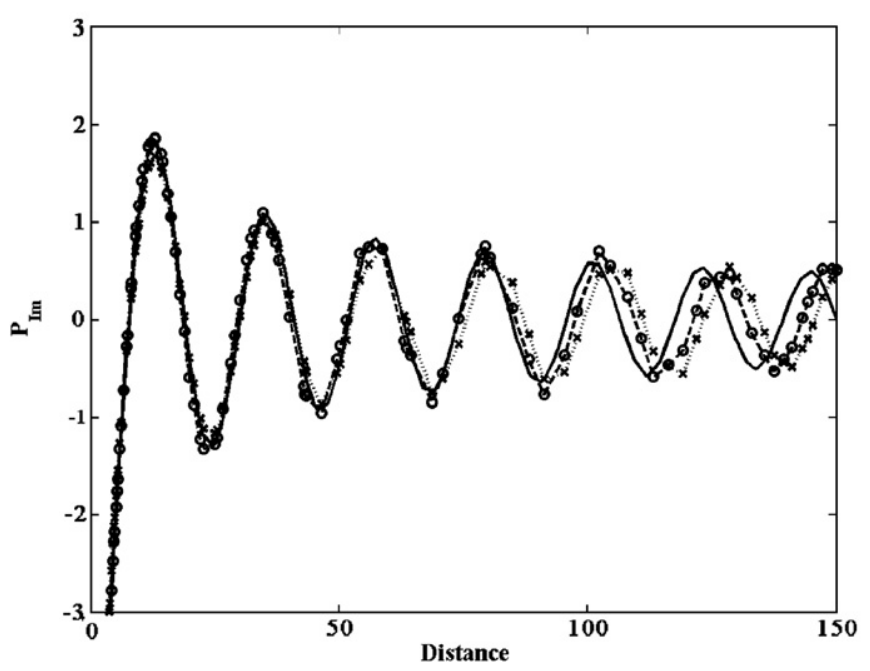

b) Imaginary part of the reference, Galerkin and SGS solutions

for a one dimensional cut of the domain at $135^{\circ}$ with respect to the $\mathrm{x}$-axis.

Continuous line: reference solution, Dashed with circles: ASGS, Dotted cross: Galerkin.

Fig. 14. Imaginary part of the solutions for one-dimensional cuts. 
cylinder propagates outwards, to the far field. In Fig. 12a, where the reference far field solution is plotted, this becomes fully evident. In Fig. 12b we have presented the results corresponding to the reference solution of the Helmholtz equation (84)-(86). By comparison with Fig. 12a, we can observe as expected that the observer being at rest with the flow sees the wave fronts bended upstream. This effect is not very strong for this example because the Mach number is not very high, but it is clearly visible e.g., near the boundaries of the domain. While the solution in Fig. 12b is totally symmetric this is not the case for the solution in Fig. 12a.

In Fig. 13a and b we have respectively plotted the Galerkin solution and SGS solution corresponding to the coarse mesh case. It can be observed that the Galerkin solution presents pollution error, which manifests as a phase lag in the wave fronts of Fig. 13a when compared with those of the reference solution in Fig. 12a. This phase error is considerably reduced when using the SGS stabilization, see Fig. 13b. The situation becomes more apparent in Fig. 14a where the results for a one-dimensional cut of the domain $\left.\Omega_{\mathrm{ac}}\right|_{\theta=90^{\circ}}=\left\{\left(r, 90^{\circ}\right) \mid D / 2<r<1.5 \times 10^{3}\right\}$ are given. It can be seen that the ASGS stabilized solution has almost the same phase as the reference one, while the Galerkin solution clearly presents a phase lag. In Fig. 14b, we give the results for another one-dimensional cut, $\left.\Omega_{\mathrm{ac}}\right|_{\theta=135^{\circ}}=\left\{\left(r, 135^{\circ}\right) \mid D / 2<r<1.5 \times 10^{3}\right\}$, which is near the limits of the silent cone in front of the cylinder. In this case the ASGS stabilization is not able to fully match the reference solution although it improves the results from the Galerkin one. From Figs. 13 and 14 we can then conclude that the ASGS stabilization yields a solution much more prone to the reference one than the Galerkin solution.

The results for the ASGS solution in this section have been obtained using an angle of $\theta=0^{\circ}$ in the effective wavenumber components (62), (63), to be inserted in the expression (46) for the stabilization parameter, $\tau_{\mathrm{SGS}}$.

\section{Conclusions}

In this paper we have presented an algebraic subgrid scale finite element method to solve the two-dimensional convected Helmholtz equation. The method does in fact correspond to the application of the Galerkin/LeastSquares approach with appropriate redefinition of its stabilization parameter, because the involved convected Helmholtz differential operator is selfadjoint. The stabilization parameter has been derived from a dispersion analysis and it reduces to well-known expressions for the onedimensional and two-dimensional Helmholtz equations in the case of no convection.

As a first application, we have considered the case of a plane wave propagating in a uniform flow. It has been shown that the proposed subgrid scale stabilized method yields exact nodal solutions, while the Galerkin method is unable to give a valid solution for the plane wave. Improvements have been also obtained for non-structured meshes different to the one used to derive the stabilization parameter and for waves propagating in different directions with respect to the mean flow. As a second example, we have considered the case of aerodynamic sound generated by flow past a two-dimensional cylinder. We have limited to subsonic cases and made use of Lighthill's acoustic analogy. Although a non-structured mesh of triangular elements has been used for this problem, it has been shown that using the subgrid scale stabilization to find the solution of the convected Helmholtz equation clearly improves the results that otherwise would have been obtained using the Galerkin finite element method.

As a general conclusion we may say, on one hand, that the herein presented ASGS stabilized method could prove very useful for problems where we have a certain degree of a priori information on the wave propagation directions. This would be the case, for instance, of duct acoustics (note that in this case two optimum stabilization parameters respectively accounting for upstream and downstream waves could be easily implemented). On the other hand, we have also checked that the method could also be useful in much more involved problems such as the generation of aeolian tones described above.

\section{References}

[1] I.M. Babuška, F. Ihlenburg, E. Paik, S.A. Sauter, A generalized finite element method for solving the Helmholtz equation in two dimensions with minimal pollution, Comput. Methods Appl. Mech. Engrg. 128 (1995) 325-359.

[2] I.M. Babuška, J.E. Osborn, Generalized finite element methods: their performance and their relation to mixed methods, SIAM J. Numer. Anal. 20 (3) (1983) 510-536.

[3] I.M. Babuška, S.A. Sauter, Is the pollution effect of the FEM avoidable for the Helmholtz equation considering high wave numbers, SIAM J. Numer. Anal. 34 (6) (1997) 2392-2423.

[4] J. Cipolla, Subgrid modeling in a Galerkin method for the Helmholtz equation, Comput. Methods Appl. Mech. Engrg. 177 (1998) 35-49.

[5] R. Codina, Comparison of some finite element methods for solving the diffusion-convection-reaction equation, Comput. Methods Appl. Mech. Engrg. 156 (1998) 185-210.

[6] R. Codina, Stabilized finite element approximation of transient incompressible flows using orthogonal subscales, Comput. Methods Appl. Mech. Engrg. 191 (2002) 4295-4321.

[7] R. Codina, J. Principe, O. Guasch, S. Badia, Time dependent subscales in the stabilized finite element approximation of incompressible flow problems, Comput. Methods Appl. Mech. Engrg. 196 (21-24) (2007) 2413-2430.

[8] D. Crighton, Computational aeroacoustics for low Mach number flows, in: J. Hardin, M. Hussaini (Eds.), Computational Aeroacoustics, Springer-Verlag, 1993.

[9] S. Crow, Aerodynamic sound emission as a singular perturbation problem, Stud. Appl. Math. 49 (1) (1970) 21-44.

[10] A. Deraemaeker, I. Babuška, P. Bouillard, Dispersion and pollution of the FEM solution for the Helmholtz equation in one, two and three dimensions, Int. J. Numer. Methods Engrg. 46 (1999) 471-499.

[11] P. Drazin, Introduction to Hydrodynamic Stability, Cambridge Texts in Applied Mathematics, Cambridge University Press, 2002.

[12] C. Farhat, I. Harari, L. Franca, The discontinuous enrichment method, Comput. Methods Appl. Mech. Engrg. 190 (2001) 6455-6479.

[13] L. Franca, A. Macedo, A two-level finite element method and its application to the Helmholtz equation, Int. J. Numer. Methods Engrg. 43 (1998) 23-32. 
[14] L. Franca, F. Valentin, On an improved unusual stabilized finite element method for the advective-reactive-diffusive equation, Comput. Methods Appl. Mech. Engrg. 190 (2000) 1785-1800.

[15] G. Gabard, R. Astley, M. Tahar, Stability and accuracy of finite element methods for flow acoustics I: General theory and application to one-dimensional propagation, Int. J. Numer. Methods Engrg. 63 (7) (2005) 947-973.

[16] G. Gabard, R. Astley, M. Tahar, Stability and accuracy of finite element methods for flow acoustics II: Two-dimensional effects, Int. J. Numer. Methods Engrg. 63 (7) (2005) 974-987.

[17] M.E. Goldstein, Aeroacoustics, McGraw-Hill, 1976.

[18] S. Goldstein, Modern Developments in Fluid Mechanics, vol. 2, Dover, New York, 1965.

[19] O. Guasch, R. Codina, Cálculo del ruido aerodinámico generado por el flujo de aire alrededor de un cuerpo. Simulación mediante métodos estabilizados de elementos finitos, in: C.M. Soares et al. (Eds.), Proc. Métodos Computacionais em Engenharia, APMTAC-SEMNI, Laboratório Nacional de Engenharia Civil de Lisboa, 2004, ISBN 972-492008-9.

[20] I. Harari, A survey of finite element methods for time harmonic acoustics, Comput. Methods Appl. Mech. Engrg. 195 (2006) 1594 1607.

[21] I. Harari, T. Hughes, Finite element methods for the Helmholtz equation in an exterior domain: model problems, Comput. Methods Appl. Mech. Engrg. 87 (1991) 57-96.

[22] I. Harari, T. Hughes, Galerkin/least-squares finite element methods for the reduced wave equation with non-reflecting boundary conditions in unbounded domains, Comput. Methods Appl. Mech. Engrg. 98 (3) (1992) 411-454.

[23] I. Harari, F. Magoulès, Numerical investigations of stabilized finite element computations for acoustics, Wave Motion 39 (2004) 339-349.

[24] I. Harari, C. Nogueira, Reducing dispersion of linear triangular elements for the Helmholtz equation, ASCE J. Eng. Mech. 128 (3) (2002) 351-358.

[25] G. Hauke, A simple subgrid scale stabilized method for the advection-diffusion-reaction equation, Comput. Methods Appl. Mech. Engrg. 191 (2002) 2925-2947.

[26] M.S. Howe, Acoustics of Fluid-Structure Interactions, Cambridge University Press, 1998.

[27] T. Hughes, Multiscale phenomena: Green's function, the Dirichlet-toNeumann formulation, subgrid scale models, bubbles and the origins of stabilized formulations, Comput. Methods Appl. Mech. Engrg. 127 (1995) 387-401.

[28] T. Hughes, L. Franca, M. Balestra, A new finite element formulation for computational fluid dynamics: V. Circumventing the BabuškaBrezzi condition: a stable Petrov-Galerkin formulation for the Stokes problem accommodating equal-order interpolations, Comput. Methods Appl. Mech. Engrg. 59 (1986) 85-99.

[29] F. Ihlenburg, Finite Element Analysis of Acoustic Scattering, Applied Mathematical Sciences, vol. 132, Springer-Verlag, 1998
[30] F. Ihlenburg, I.M. Babuska, Finite element solution to the Helmholtz equation with high wave number. Part I: The $h$-version of the FEM, Comput. Math. Appl. 39 (1995) 9-37.

[31] O. Inoue, N. Hatakeyama, Sound generation by a two-dimensional circular cylinder in a uniform flow, J. Fluid Mech. 471 (2002) 285-314.

[32] R. Kechroud, A. Soulaimani, Y. Saad, S. Gowda, Preconditioning techniques for the solution of the Helmholtz equation by the finite element method, Math. Comput. Simul. 65 (4-5) (2004) 303-321.

[33] O. Laghrouche, P. Bettess, Short wave modelling using special finite element method, J. Comput. Acoust. 8 (1) (2000) 189-210.

[34] M. Lighthill, On sound generated aerodinamically I. General theory, Proc. R. Soc. Lond. 211 A 1107 (1952) 564-587.

[35] J. Melenk, I. Babuska, The partition of unity method: basic theory and applications, Comput. Methods Appl. Mech. Engrg. 139 (1-4) (1996) 289-314.

[36] S. Mittal, V. Kumar, Flow-induced vibrations of a light circular cylinder at Reynolds numbers $10^{3}-10^{4}$, J. Sound Vib. 245 (5) (2001) 923-946.

[37] A. Oberai, P. Pinsky, A multiscale finite element method for the Helmholtz equation, Comput. Methods Appl. Mech. Engrg. 154 (1998) 3281-3297.

[38] A. Oberai, P. Pinsky, A numerical comparison of finite element methods for the Helmholtz equation, J. Comput. Acoust. 8 (1) (2000) 211-221.

[39] A. Oberai, P. Pinsky, A residual-based finite element method for the Helmholtz equation, Int. J. Numer. Methods Engrg. 49 (2000) 399419.

[40] A. Oberai, F. Roknaldin, T. Hughes, Computational procedures for determining structural-response due to hydrodynamic sources, Comput. Methods Appl. Mech. Engrg. 190 (2000) 345-361.

[41] A. Oberai, F. Roknaldin, T. Hughes, Computation of trailing-edge noise due to turbulent flow over an airfoil, AIAA J. 40 (11) (2002) 2206-2216.

[42] O. Phillips, The intensity of aeolian tones, J. Fluid Mech. 1 (1956) 607-624.

[43] J. Ristorcelli, A closure for the compressibility of the source terms in Lighthill's acoustic analogy, Technical Report ICASE Report 97-44, NASA/CR-1997-201738, 1997.

[44] S. Slimon, M. Soteriou, D. Davis, Development of computational aeroacoustics equations for subsonic flows using a Mach number expansion approach, J. Comput. Phys. 159 (2000) 377-406.

[45] J. Stewart, T. Hughes, $h$-adaptative finite element computation of time-harmonic exterior acoustics problems in two dimensions, Comput. Methods Appl. Mech. Engrg. 146 (1-2) (1997) 65-89.

[46] L. Thompson, P. Pinsky, A Galerkin least-squares finite element method for the two-dimensional Helmholtz equation, Int. J. Numer. Methods Engrg. 38 (1995) 371-397.

[47] L. Thompson, P. Pinsky, Acoustics, in: E. Stein, R. de Borst, T.J.R. Hughes (Eds.), Encyclopedia of Computational Mechanics, Wiley, 2004. 